\title{
19 Ökonomie und globale Gesundheit
}

\author{
Stefan Kohler, Max Roser, Pascal Geldsetzer, Till Bärnighausen
}

\subsection{Einleitung}

Wohlstand, Gesundheit und Gesundheitsausgaben sind eng miteinander verknüpft. Im weltweiten Durchschnitt haben alle drei seit vielen Jahren stetig zugenommen. Im Vergleich haben Menschen in Ländern mit höheren Einkommen eine höhere Lebenserwartung. Die höchste Krankheitslast pro Mensch tritt in Ländern mit niedrigem Einkommen auf. Den größten Anteil an der gesamten globalen Krankheitslast haben Länder mit mittlerem Einkommen, in denen rund drei Viertel der Weltbevölkerung lebt. Im Mittel sind die Gesundheitsausgaben in Ländern mit höheren Einkommen insgesamt sowie pro Kopf höher als die Gesundheitsausgaben in Ländern mit niedrigeren Einkommen. Die größten Zuwachsraten bei Einkommen und Gesundheit hatten in den letzten Jahrzehnten Menschen in Ländern mit niedrigen und mittleren Einkommen. Die seit dem 19. Jahrhundert stark angewachsene Einkommensungleichheit zwischen Ländern nahm deswegen seit etwa Mitte der 1990er Jahre ab.

Neben dem Gesundheitszustand der Bevölkerung und der Höhe der Gesundheitsausgaben unterscheidet sich auch die Finanzierung der Gesundheitsausgaben mit dem Wohlstandsniveau. Der Großteil der Gesundheitsausgaben in Ländern mit hohem oder oberem mittlerem Einkommen ist durch Steuern oder Krankenversicherungsbeiträge finanziert. Diese vom Eintreten eines Krankheitsfalls entkoppelte Gesundheitsfinanzierung schützt vor ruinösen finanziellen Folgen von Krankheit. In Ländern mit niedrigem oder niedrigem mittlerem Einkommen hingegen wird mehr als ein Drittel der Gesundheitsausgaben im Krankheitsfall aus eigener Tasche bezahlt. Bevor mit zunehmendem Wohlstand ein Übergang weg von Selbstzahlungen im Krankheitsfall hin zu einer Gesundheitsfinanzierung durch Steuern und Versicherungsbeiträge stattfindet, nimmt der Anteil von Selbstzahlungen an den Gesundheitsausgaben eines Landes zunächst zu. Ein Grund dafür ist die geringere Unterstützung durch Entwicklungshilfe für Gesundheit in Ländern mit mittlerem Einkommen im Vergleich zu Ländern mit niedrigem Einkommen.

Dieses Kapitel führt in Zusammenhänge zwischen Wohlstand, Gesundheit und Gesundheitsausgaben sowie deren weltweite Entwicklung ein. Globale Daten zu Wohlstand, Gesundheit und Gesundheitsausgaben, die als Weltentwicklungsindikatoren oder im Rahmen der Global Burden of Disease Studie und der Financing Global Health Studie regelmäßig erhoben werden, werden vorgestellt und besprochen. Aktuelle globale Unterschiede werden mit historischen Wirtschafts- und Gesundheitsdaten aus Deutschland verglichen. Auswirkungen der COVID-19-Pandemie auf Trends der letzten Jahre werden angesprochen. 


\subsection{Wohlstand und Gesundheit im internationalen Vergleich}

\subsubsection{Zusammenhang zwischen Wohlstand und Lebenserwartung}

Es gibt verschiedene Wohlstandsindikatoren. In diesem Kapitel wird als Näherungswert für das mittlere Wohlstandsniveau in einem Land das Bruttoinlandsprodukt pro Kopf herangezogen, das auch als mittleres Einkommen gedeutet wird. In Abb. 19.1 sind Bruttoinlandsprodukt pro Kopf und die Lebenserwartung bei Geburt als Maß für Gesundheit für die Jahre 2019 und 1990 im globalen Vergleich dargestellt. Jedes Land, für das Daten zu Lebenserwartung, Bruttoinlandsprodukt und Bevölkerungszahl als Weltentwicklungsindikatoren (World Development Indicators) vorlagen, ist als Kreis dargestellt, dessen Fläche proportional zur Bevölkerungszahl ist. Neben dem globalen Vergleich wird die historische Entwicklung in Deutschland gezeigt.

Um das Bruttoinlandsprodukt pro Kopf im zeitlichen Verlauf und zwischen Ländern vergleichen zu können, wird dieses in internationalen Dollar und 2017 Preisen ausgedrückt. Die Kaufkraft eines internationalen Dollar entspricht der Kaufkraft eines US-Dollar in den Vereinigten Staaten zum angegebenen Zeitpunkt. Statt internationalen Dollar wird deshalb auch von kaufkraftbereinigten US-Dollar gesprochen. Mittels dieser nicht realen Währungseinheit und dem Bezug auf ein Referenzjahr werden Vergleiche von Bruttoinlandsprodukt oder Einkommen zwischen Ländern und über die Zeit näherungsweise um Kaufkraftunterschiede und Inflation bereinigt. Ein internationaler Dollar sollte, nach Umrechnung in Landeswährung, ermöglichen in jedem Land eine vergleichbare Menge an Waren und Dienstleistungen zu kaufen.

Menschen, die in einem wohlhabenderen Land geboren sind, leben im Durchschnitt länger als in ärmeren Ländern geborene Menschen. Der Zusammenhang zwischen einer Veränderung des wirtschaftlichen Wohlstands und einer Veränderung in der Lebenserwartung ist für wirtschaftlich schwächere Länder stärker ausgeprägt und nimmt mit zunehmendem Wohlstand ab. Dies bedeutet, dass in der Gruppe der Länder mit niedrigem Einkommen (s. Kasten Ländereinteilung am Ende des Abschnitts) eine Wohlstandssteigerung mit einer vergleichsweise starken möglichen Zunahme der Lebenserwartung in Verbindung gebracht wird. Mit zunehmendem Wohlstandsniveau geht jede weitere Wohlstandssteigerung jeweils mit geringeren Steigerungen der Lebenserwartung einher.

Das durchschnittliche Verhältnis zwischen Bruttoinlandsprodukt pro Kopf und Lebenserwartung, die sogenannte Preston Kurve, ist in Abb. 19.1 näherungsweise als logarithmische Kurve bestimmt. Diese Kurve wurde durch eine Regressionsanalyse an die Daten angepasst und ist nach dem amerikanischen Demografen und Soziologen Samuel H. Preston, dem Erstbeschreiber der Beziehung zwischen Wohlstand und Gesundheit im internationalen Vergleich, benannt [1]. Jedes einzelne Land kann über oder unter dieser Kurve liegen. Jene unterhalb der Preston Kurve, wie beispielsweise die USA, Saudi-Arabien, Russland oder Südafrika, haben Lebenserwartungsniveaus, die niedriger sind als die Lebenserwartung, die allein auf der Grundlage des 


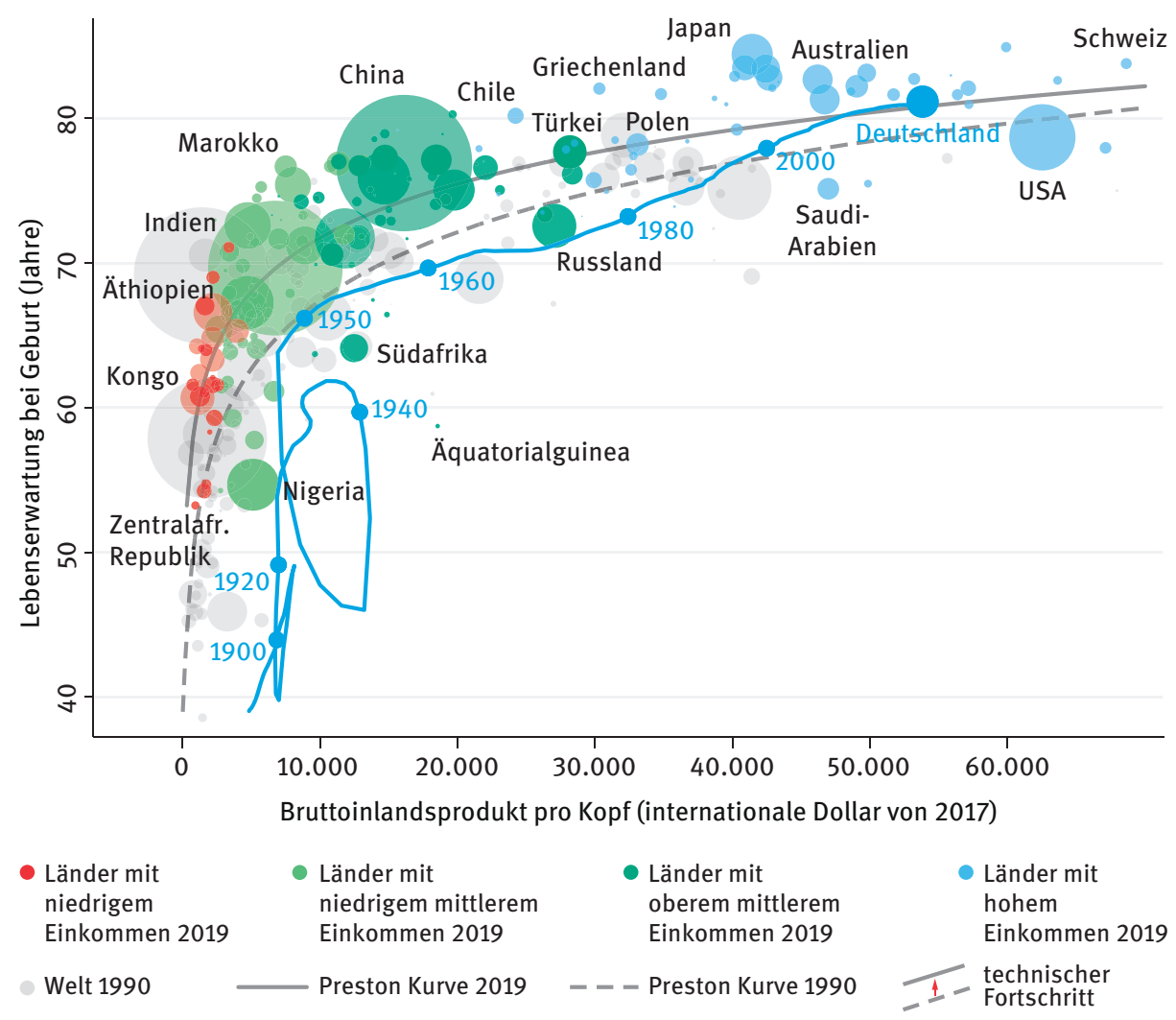

Abb. 19.1: Lebenserwartung und Bruttoinlandsprodukt pro Kopf weltweit in den Jahren 1990 und 2019 und in Deutschland seit 1900. Die Kreisfläche ist proportional zur Bevölkerungsverteilung im jeweiligen Jahr. Logarithmische Preston Kurven wurden ohne Gewichtung hinsichtlich der Bevölkerungszahl ermittelt. Länder mit einem Bruttoinlandsprodukt pro Kopf > 70.000 internationale Dollar von 2017 sind nicht dargestellt. Rwanda mit der weltweit niedrigsten Lebenserwartung von 33 Jahren im Jahr 1990 ist nicht dargestellt. Für das Jahr 2019 waren Daten für 173 Länder und Gebiete verfügbar, für das Jahr 1990 für 161. Historische Lebenserwartung und Bruttoinlandsprodukt in Deutschland seit 1900 sind als Fünfjahresdurchschnitt dargestellt. Das Bruttoinlandsprodukt pro Kopf für Deutschland bis 1989 wurde auf Basis des Verhältnisses zwischen internationalen Dollar von 2011 und 2017 in Deutschland im Jahr 1990 in internationale Dollar von 2017 umgerechnet. Datenquellen: Gapminder und World Development Indicators.

wirtschaftlichen Wohlstands vorhergesagt würde. Länder über der Kurve, wie Äthiopien, Marokko, China oder Japan, haben mittlere Lebenserwartungen, die für die gegebene wirtschaftliche Entwicklung überdurchschnittlich hoch sind.

Am ältesten wurde im Landesdurchschnitt über viele Jahre hinweg die Bevölkerung Japans. Da die Lebenserwartung in Japan höher ist als die Lebenserwartung in vergleichbar wohlhabenden Ländern, liegt Japan mit einer geschätzten Lebenserwar- 
tung von 84 Jahren im Jahr 2019 deutlich oberhalb der angepassten Kurve. Je nach Datenquelle folgen die Schweiz, Spanien, Italien und Island beziehungsweise folgt oder überholte bereits Singapur (mit einem Pro-Kopf-Bruttoinlandsprodukt von 97.341 internationalen Dollar von 2017 nicht in Abb. 19.1). Berücksichtigt man zudem Gebiete, für die gesonderte World Development Indicators vorliegen, dann war die geschätzte Lebenserwartung in Hong Kong mit 85 Jahren im Jahr 2019 am höchsten.

Um die Entwicklung der Beziehung der Durchschnittswerte von Lebenserwartung und Wohlstand im zeitlichen Verlauf zu verdeutlichen, stellt Abb. 19.1 Lebenserwartungen und Bruttoinlandsprodukte für verschiedene Jahre dar. Im Zeitraum von 1990 bis 2019 ist das weltweite Bruttoinlandsprodukt pro Kopf von 9.661 auf 16.944 internationale Dollar von 2017 gestiegen und die weltweite Lebenserwartung hat von 65 Jahre auf 73 Jahre zugenommen. Aus dem Vergleich der farbigen Kreise für das Jahr 2019 mit den darunterliegenden grauen Kreisen für das Jahr 1990 lässt sich Folgendes erkennen:

1. Vom Zuwachs an Lebenserwartung und Einkommen haben die meisten Länder profitiert - in 150 von 153 Volkswirtschaften mit Daten für die Jahre 1990 und 2019 sind beide angestiegen.

2. Die weltweiten Veränderungen, die viele Menschen betreffen, werden stark durch die Entwicklung der bevölkerungsreichen Länder China und Indien beeinflusst - in diesen beiden Ländern lebten 43 \% der Weltbevölkerung im Jahr 1990 und $36 \%$ der Weltbevölkerung im Jahr 2019.

3. Die weltweit geringsten Lebenserwartungen sind angestiegen - von 33 Jahre in Rwanda, 39 Jahre in Sierra Leone und 44 Jahre in Südsudan im Jahr 1990 auf 53 Jahre in der Zentralafrikanischen Republik, 54 Jahre im Chad und in Lesotho sowie 55 Jahre in Nigeria und Sierra Leone im Jahr 2019.

Eine beständige weltweite Zunahme der Lebenserwartung sowie der höchsten Lebenserwartung in der Welt, wird auch für die Zukunft erwartet. Südkorea, mit einer Lebenserwartung von 83 Jahren im Jahr 2019, ist möglicherweise das Land, in dem zuerst eine durchschnittliche Lebenserwartung von über 90 Jahren erreicht wird [2].

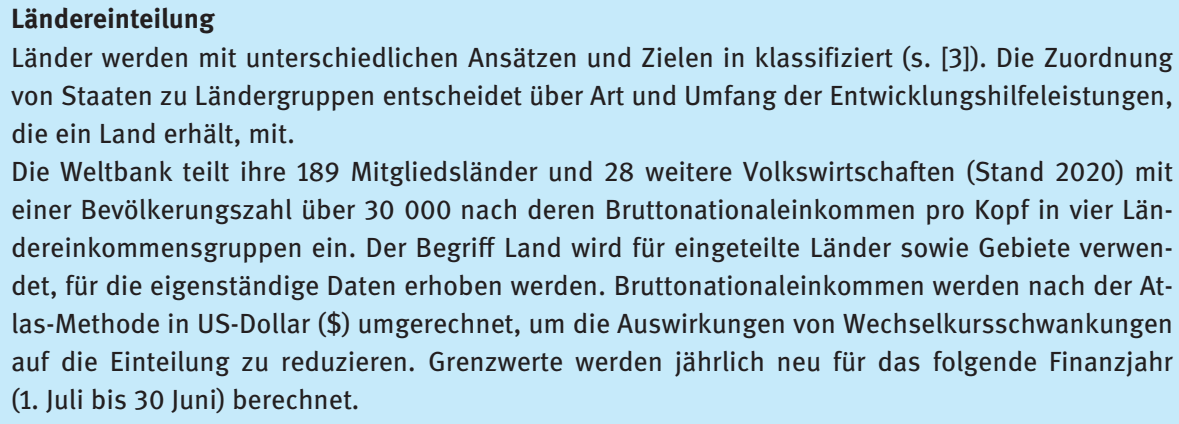




\begin{tabular}{lll}
\hline $\begin{array}{l}\text { Ländereinkommensgruppen der Weltbank } \\
\text { (World Bank Country and Lending Groups) }\end{array}$ & $\begin{array}{l}\text { Bruttonationaleinkommen pro Kopf (Anzahl } \\
\text { der Länder) } \\
\text { 2020/21 (217) }\end{array}$ & 1990/91 (167) \\
\hline $\begin{array}{l}\text { Länder mit niedrigem Einkommen } \\
\text { (Low Income Countries, LICs) }\end{array}$ & $\leq 1.035$ \$ (29) & $\leq 610$ \$(49) \\
$\begin{array}{l}\text { Länder mit niedrigem mittlerem Einkommen } \\
\text { (Lower Middle-Income Countries, LMICs) }\end{array}$ & $1.036-4.045 \$(50)$ & $611-2.465 \$(53)$ \\
$\begin{array}{l}\text { Länder mit oberem mittlerem Einkommen } \\
\text { (Upper Middle-Income Countries, UMICs) }\end{array}$ & $4.046-12.535 \$(56)$ & $2.466-7.620 \$(22)$ \\
$\begin{array}{l}\text { Länder mit hohem Einkommen } \\
\text { (High Income Countries, HICs) }\end{array}$ & $>12.535 \$(82)$ & $>7.620 \$(43)$ \\
\hline
\end{tabular}

Datenquelle: World Bank Country and Lending Groups.

\subsubsection{Medizinisch-technischer Fortschritt und Lebenserwartung}

Die Preston Kurve verschob sich in der Vergangenheit beständig nach oben. In Abb. 19.1 ist diese Entwicklung beispielhaft für die Jahre 1990 und 2019 aufgezeigt. Die Verschiebung der Preston Kurve nach oben bedeutet, dass die durchschnittliche Lebenserwartung in der Welt unabhängig von der Veränderung des Wohlstands im Verlauf der Zeit angestiegen ist. Die wohlstandsunabhängige Verbesserung der Gesundheit von Menschen weltweit wird auf medizinisch-technischen Fortschritt zurückgeführt, welcher ermöglicht bei einem vergleichbaren Wohlstandsniveau mit den verfügbaren Mitteln, heute im Durchschnitt einen besseren Gesundheitszustand zu erreichen als früher.

Gegen Ende des 19. Jahrhunderts und in der ersten Hälfte des 20. Jahrhunderts vollzog sich durch medizinisch-technischen Fortschritt eine beispiellose Wirksamkeitssteigerung bei den möglichen Maßnahmen zum Erhalt und der Wiederherstellung der Gesundheit: Wissenszuwachs in den Bereichen Krankheitsentstehung und Krankheitsverhütung ermöglichte wirksame Hygienemaßnahmen und Impfungen. Die Entdeckung von Antibiotikatherapie brachte Fortschritte bei der Krankheitsbehandlung. Die allgemeinen Wohnbedingungen wurden besser. Zudem begann zwischen den 1950er und späten 1960er Jahren die Entwicklung landwirtschaftlicher Hochertragssorten und deren Verbreitung, welche in Verbindung mit dem Einsatz von Mineraldüngern, Pestiziden und Bewässerung, in vielen Ländern die Ernährungssituation verbessert haben; eine Veränderung, die auch als Grüne Revolution bezeichnet wird [4]. 
Da global in Folge des Zusammenspiels dieser Entdeckungen insbesondere Infektionskrankheiten, welche lange Zeit die vorherrschende Todesursache darstellten, wirksamer verhindert und bekämpft werden konnten, stieg weltweit die Lebenserwartung für große Teile der Bevölkerung im Verlauf des 20. Jahrhunderts stetig an. Im Vereinigten Königreich oder in Deutschland sank beispielsweise zunächst die Kindersterblichkeit und dann auch die Sterblichkeit für einen großen Teil der Bevölkerung im mittleren Lebensalter [5,6]. Für einen kleinen Bevölkerungsteil mit besonderer Stellung in der Gesellschaft begann die stetige Zunahme der Lebenserwartung bereits früher, schätzungsweise seit Mitte des 17. Jahrhunderts [7].

Obwohl die Gesundheit ganzer Bevölkerungen oder großer Bevölkerungsteile wiederkehrend von neuen Erkrankungen, Krankheitsverläufen und Krankheitsursachen bedroht wird, setzt sich der Anstieg der Lebenserwartung voraussichtlich weltweit fort [2,8]. Seit Ende des 20. Jahrhunderts verursachen zum Beispiel nichtübertragbare Krankheiten (z. B. Herz-Kreislauf-Erkrankungen, Krebs oder psychische Störungen), neue Infektionskrankheiten (z. B. HIV/AIDS oder COVID-19) und veränderte Erreger (z. B. Antibiotika-resistente Bakterien) in großem Umfang neuartige Krankheitslast und Todesfälle [9-11]. Auch Kriege und Völkermord führen weiterhin zu einem Rückgang der durchschnittlichen Lebenserwartung in einigen Länder. Bislang hat die Lebenserwartung dennoch weltweit fortdauernd zugenommen, wenn längere Zeitspannen betrachtet werden. Gründe dafür sind unter anderem:

- ein vorübergehender Charakter gesundheitsgefährdender Ereignisse,

- der Auf- und Ausbau medizinischer Versorgung und/oder

- anhaltender medizinisch-technischer Fortschritt.

Nachfolgender Kasten schildert, am Beispiel von Südafrika, wie sich die Lebenserwartung im Zuge der Ausbreitung von HIV/AIDS, der Entdeckung einer wirksamen Therapie und deren darauffolgendem Scale-up, das heißt dem Ausbau der Therapiemöglichkeiten in großem Maßstab, entwickelt hat.

\section{Lebenserwartung, HIV/AIDS-Epidemie und antiretrovirale Therapie in Südafrika}

1960-1992 Die durchschnittliche Lebenserwartung steigt von 48 auf 63 Jahre in Südafrika und von 53 auf 66 Jahre weltweit.

1992-2005 Aufgrund der schnellen Ausbreitung von HIV-Infektionen entsteht eine HIV/AIDSEpidemie, in deren Folge die durchschnittliche Lebenserwartung der Bevölkerung Südafrikas von 63 Jahre auf 53 Jahre abnimmt.

1996 Mit der Entdeckung einer Kombinationstherapie aus mindestens drei verschiedenen antiretroviralen Medikamenten zur Behandlung der HIV-Infektion steigt die individuelle Lebenserwartung von Menschen, die mit HIV leben und antiretrovirale Therapie erhalten, stark an.

Seit 2000 In Folge des Rückgangs neuer HIV Fälle sowie des Auf- und Ausbaus antiretroviraler Therapiemöglichkeiten in Südafrika, durch die ein zunehmender Teil der Bevölkerung kostenfreien Zugang zu HIV Testung und Behandlung erhält, gehen die HIV/ AIDS-bedingten Todesfälle deutlich zurück [12,13]. 


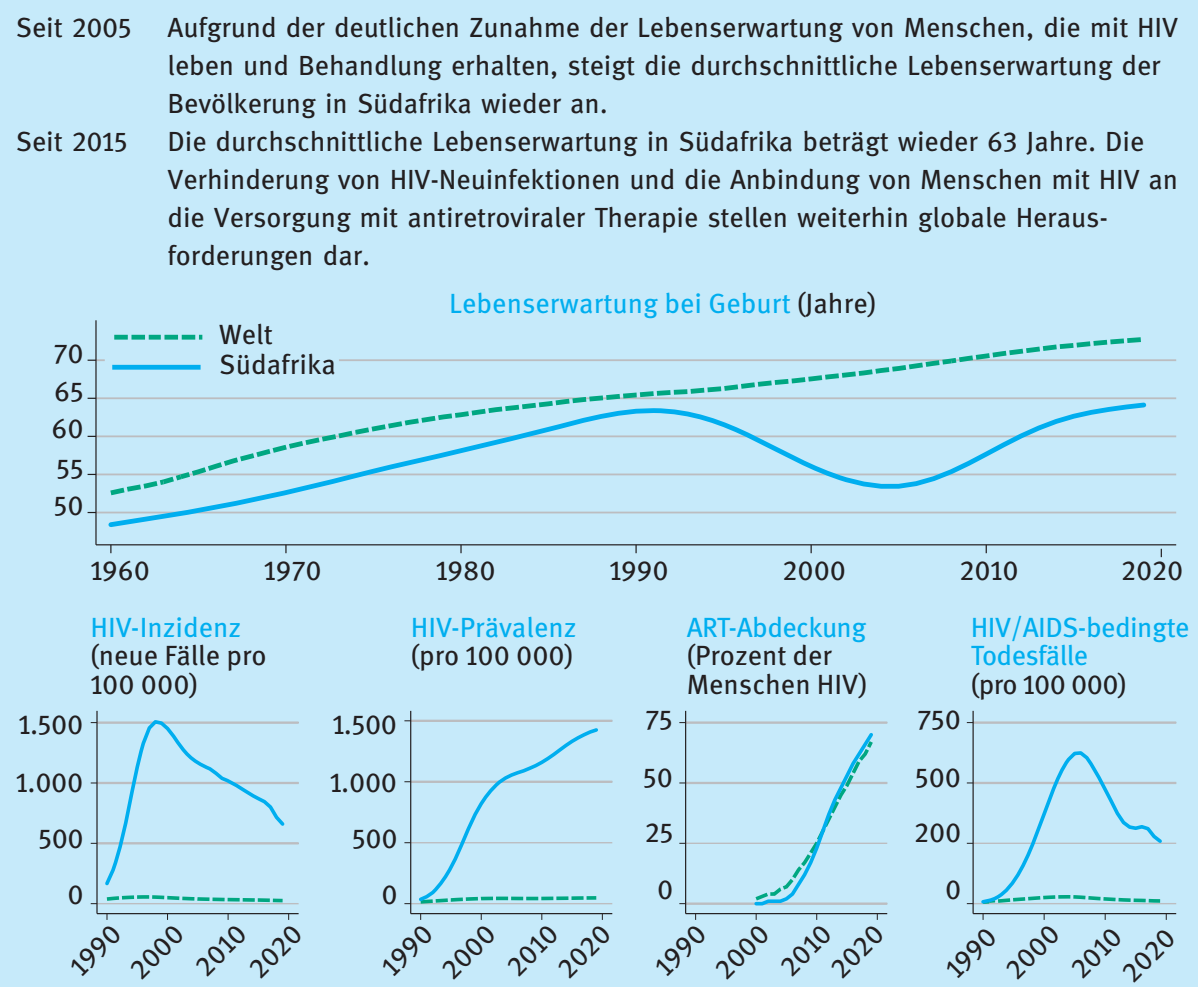

Datenquellen: Global Burden of Disease Studie 2019 und World Development Indicators.

Von Samuel H. Preston wurde geschätzt, dass einkommensunabhängige Veränderungen, wie medizinisch-technischer Fortschritt in Form von Aufwärtsverschiebungen der Preston Kurve, in der Vergangenheit 75 \% bis 90 \% des Anstiegs der Lebenserwartung zwischen 1930 und 1960 ausmachten. Einkommenswachstum (,Bewegung entlang der Preston Kurve“) wären für die restlichen $10 \%$ bis $25 \%$ des beobachteten Anstiegs der Lebenserwartung verantwortlich [1]. Eine andere Studie, welche Daten seit 1800 betrachtet, folgert wiederum, dass in der Gesamtsicht das Wohlstandswachstum den stärksten Einfluss auf die Steigerung der Lebenserwartung auf der ganzen Welt hatte. Obwohl medizinische Innovationen die Lebenserwartung erhöhen, erscheine es wahrscheinlicher, dass wohlhabendere Länder Innovationen einführen und Behandlungen durchführen, da diese dafür bezahlen können [14]. Wie diese verschiedenen Erklärungen unterschiedlicher Untersuchungen zu den Ursachen des beobachteten Zusammenhangs zwischen Wohlstand und Gesundheit veranschaulichen ist umstritten, welche Ursache-Wirkungs-Zusammenhänge der Preston Kurve und ihren Veränderungen in welchem Umfang unterliegen. Die Gründe hierfür werden in Kap. 19.2.4 näher besprochen. 


\subsubsection{Historische Entwicklung von Wohlstand und Lebenserwartung in Deutschland}

Die historische Entwicklung von Einkommen und Lebenserwartung in Deutschland ist ebenfalls in Abb. 19.1 veranschaulicht. Im Jahr 1900 betrug die Lebenserwartung in Deutschland schätzungsweise 44 Jahre; 46 Jahre für Frauen und 43 Jahre für Männer (s. [15]). Damit lag die Lebenserwartung in Deutschland im Jahr 1900 unter der geringsten Lebenserwartung aller Länder im Jahr 2019, welche auf 53 Jahre in der Zentralafrikanischen Republik geschätzt wurde. Der stärkste Anstieg der durchschnittlichen Lebenserwartung in Deutschland vollzog sich in der ersten Hälfte des 20. Jahrhunderts bei einem vergleichsweise geringem Anstieg des Bruttoinlandsprodukts pro Kopf während dieses Zeitraums. Im weiteren Verlauf stieg das Bruttoinlandsprodukt stärker an, die Lebenserwartung allerdings stieg im Verhältnis dazu in geringerem Maße weiter.

Die historische Entwicklung Deutschlands zeigt auf wie Wohlstand und Gesundheit in einem Land mit jetzt hohem Einkommen im Laufe von etwa 120 Jahren stark zugenommen haben. Dabei wird deutlich, dass die durchschnittlichen Niveaus von Wohlstand und Gesundheit, aus heutiger Sicht, in Ländern mit hohem Einkommen noch vor etwa vier Generationen niedrig waren. Preston Kurve und die historische Entwicklung der Lebenserwartung in Deutschland legen nahe, dass in Ländern mit niedrigen Lebenserwartungen deutliche Verbesserungen der Gesundheit möglich und, unterstützt durch technischen-Fortschritt, auch erreichbar sind.

\subsubsection{Macht Armut krank und Wohlstand gesund?}

Die Preston Kurve und das Beispiel der historischen Entwicklung von Wohlstand und Lebenserwartung in Deutschland bringen beide einen Zusammenhang zwischen der Gesundheit der Bevölkerung und der wirtschaftlichen Situation eines Landes zum Ausdruck. Zu dem Ausmaß, in dem die beobachtete Verbindung von wirtschaftlichem Wohlstand und Gesundheit auf einer Ursache-Wirkungs-Beziehung beruht, bei dem ein Wohlstandszuwachs zu mehr Gesundheit führt, könnte eine Umverteilung von Ländern mit höherem Wohlstand zu Ländern mit geringerem Wohlstand die durchschnittliche Gesundheit der Menschheit erhöhen. Falls aber der Zusammenhang zwischen Wohlstand und Lebenserwartung von äußeren Einflussfaktoren verursacht wird, also nur scheinbar eine ursächliche Beziehung zwischen Wohlstand und Gesundheit besteht oder, umgekehrt, sogar Gesundheit zu höherem Wohlstand führt, dann würde sich eine Vermögens- oder Einkommensumverteilung zwischen Ländern nicht auf die Lebenserwartung auswirken.

Der mit der Preston Kurve beschriebene Zusammenhang lässt ohne weitere Kenntnisse keine Aussage darüber zu, ob und wie eine Ursache-Wirkungs-Beziehung zwischen dem Bruttoinlandsprodukt pro Kopf und der Lebenserwartung, welche als 
Maß für die Gesundheit herangezogen wurde, besteht. Ein wohlhabenderes Land verfügt zum Beispiel über mehr Mittel, um Gesundheitsdienstleistungen zu finanzieren, welche zu einer besseren Gesundheit beitragen können. Allerdings ist es ebenso möglich, dass eine bessere Gesundheit zu höherem Einkommen führt und sich umgekehrt ein schlechterer Gesundheitszustand einer Bevölkerung nachteilig auf die wirtschaftliche Leistungsfähigkeit und damit den Wohlstand eines Landes auswirkt. Neben diesen Ursache-Wirkungs-Beziehungen werden sowohl Wohlstand als auch Gesundheit von gemeinsamen Einflussfaktoren, wie der sozialen und demographischen Zusammensetzung eines Landes, dessen Infrastruktur, Bildungschancen, Regierungsführung oder der Gesundheit in Ländern, mit denen Austauschbeziehungen bestehen, mitbestimmt. Deshalb können auch gemeinsame Einflussfaktoren eine Erklärung für gemeinsam auftretende Unterschiede bei Gesundheit und Wohlstand darstellen. Es gibt also drei plausible Erklärungen für das Vorhandensein eines Zusammenhangs zwischen Gesundheit und wirtschaftlichem Wohlstand, welche sich nicht gegenseitig ausschließen (s. Kasten und [16]).

\section{Erklärungen für den Zusammenhang zwischen Gesundheit und Wohlstand}

1. Erhöhtes Einkommen führt zu besserer Gesundheit (Kausalzusammenhang).

2. Gesündere Arbeitnehmer sind produktiver und verdienen deshalb besser (umgekehrter Kausalzusammenhang).

3. Äußere Einflussfaktoren verursachen sowohl eine bessere Gesundheit als auch einen höheren Wohlstand (Korrelation ohne Kausalzusammenhang - auch missverständlich als Scheinkorrelation bezeichnet, da nicht nur scheinbar, sondern tatsächlich eine Korrelation, jedoch keine Kausalität vorliegt).

Mehrere Erklärungen können gleichzeitig zutreffen. Dies spiegelt beispielsweise die Strategie der Bundesregierung zur globalen Gesundheit wider [17]:

„Gesundheit ist nicht nur eine Voraussetzung für Wohlstand, sozialen Zusammenhalt und gesellschaftliche Teilhabe, sondern auch Ergebnis und Indikator für die soziale, wirtschaftliche und ökologische Dimension nachhaltiger Entwicklung. “(Aus dem Vorwort)

„Zudem wird Deutschland verstärkt den Schulterschluss mit anderen Förderern vor Ort und mit den VN Country Teams [Länderteams der Vereinten Nationen] suchen, um Maßnahmen und Partnerschaften ins Leben zu rufen, welche die wirtschaftliche Entwicklung voranbringen und die Gesundheit der Bevölkerung befördern. “(Aus dem Ziel II.3 Regionale Partnerschaften ausbauen)

Da alle drei genannten Erklärungen zutreffen können, ist es schwierig, das Ausmaß der alleinigen Wirkung von Wohlstand auf Gesundheit zu ermitteln. Eine direkte Abschätzung der Auswirkung einer Veränderung des Pro-Kopf-Bruttoninlandsprodukts auf die Lebenserwartung anhand der Preston Kurve würde fälschlicherweise auch den umgekehrten Einfluss von Gesundheit auf Wohlstand und den Einfluss gemeinsamer Bestimmungsfaktoren von Wohlstand und Gesundheit beinhalten. 


\subsubsection{Folgerungen für die globale Gesundheitspolitik}

Aufgrund der verschiedenen Erklärungen für den Zusammenhang zwischen Gesundheit und Wohlstand, sollten wirtschaftliche Entwicklung und Verbesserungen der allgemeinen Lebensbedingungen zur mittel- bis langfristigen Verbesserung der globalen Gesundheit mit bedacht und unterstützt werden. Oft ist jedoch unsicher oder unbekannt in welchem Ausmaß die Gesundheit durch Wohlstand beeinflusst wird, Wohlstand Gesundheit fördert oder sonstige Umstände beides beeinflussen. Deshalb ist es in der Praxis schwierig, die zielführendste Mischung aus direkter und indirekter Gesundheitsförderung zu finden und die Förderung verschiedener Entwicklungsziele auszubalancieren. Ökonomische Bewertung, die im Kap. 20 besprochen wird, kann durch eine strukturierte Vorgehensweise helfen, Handlungsalternativen zu vergleichen und möglichst nützliche Maßnahmen zur Erreichung eines Ziels zu erkennen.

\subsection{Globale Einkommensverteilung}

\subsubsection{Entwicklung der globalen Einkommensverteilung und Ungleichheit seit $\mathbf{1 8 0 0}$}

Im vorausgehenden Abschnitt wurden Durchschnittswerte ohne Verteilungsaspekte betrachtet. Nun werden Schätzwerte für das Bruttoinlandsprodukt und seine Verteilung in einem Land herangezogen, um die Entwicklung der globalen Einkommensverteilung und Ungleichheit zu beschreiben. Abb. 19.2 zeigt die geschätzte Entwicklung der Verteilung der Tageseinkommen aller Menschen auf der Welt seit 1800 in internationalen Dollar von 2011. Im Jahr 1800 gab es in den meisten Ländern nur sehr wenige wohlhabende Menschen. Der Großteil der Menschen auf der Welt, etwa $90 \%$, lebte in wirtschaftlichen Verhältnissen, die wir heute als extreme Armut bezeichnen (s. Kasten Extreme Armut am Ende des Abschnitts).

Beginnend mit der Industrialisierung in Nordwesteuropa stiegen zunächst die Einkommen in diesem Teil der Welt. Der materielle Wohlstand im Rest der Welt blieb hingegen niedrig. Während die Industrialisierung schließlich auch in einigen nichteuropäischen Ländern erfolgte - zunächst in Nordamerika, Ozeanien, Teilen Südamerikas und später in Japan und Ostasien - blieben andere Länder in Asien und Afrika arm. In Folge nahm die globale Ungleichheit der Einkommensverteilung über einen Zeitraum von fast zwei Jahrhunderten zu. Zwischen den 1960er und 1990er Jahren wies die weltweite Einkommensverteilung zwei Häufigkeitsmaxima („Gipfel“) auf. Dies bedeutet, die Welt war, so deutlich wie nie vor und nie nach dieser Zeit, in eine ärmere Bevölkerung und eine wohlhabendere Bevölkerung geteilt. Im Jahr 1980 lag beispielsweise das weltweit häufigste Einkommen bei knapp 1 internationaler Dollar von 2011 pro Tag und das zweithäufigste Einkommen bei etwa 18 internationale Dollar von 2011 pro Tag (Abb. 19.2a-d). 


\section{(a) 1800}

982 Mio. Menschen auf der Welt

881 Mio. (90\%) leben in extremer Armut

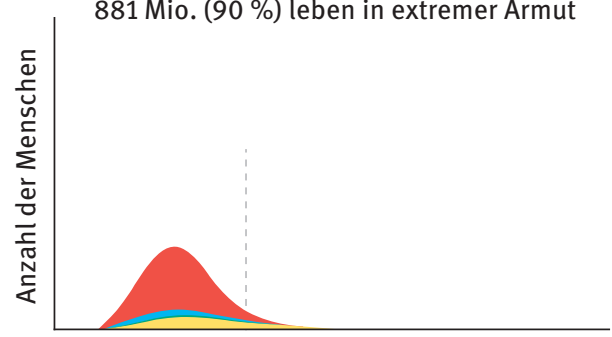

(c) 1960

3,0 Mrd. Menschen auf der Welt

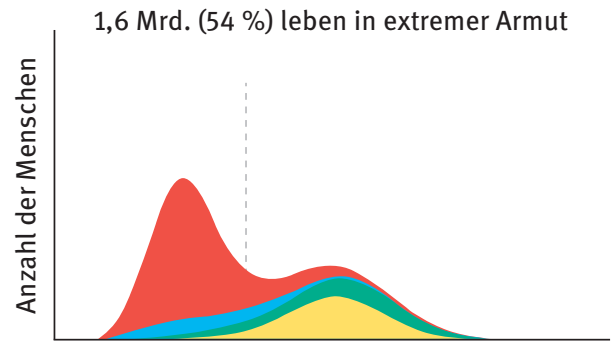

(e) 2019 Vorhersage

7,7 Mrd. Menschen auf der Welt

778 Mio. (10\%) leben in extremer Armut

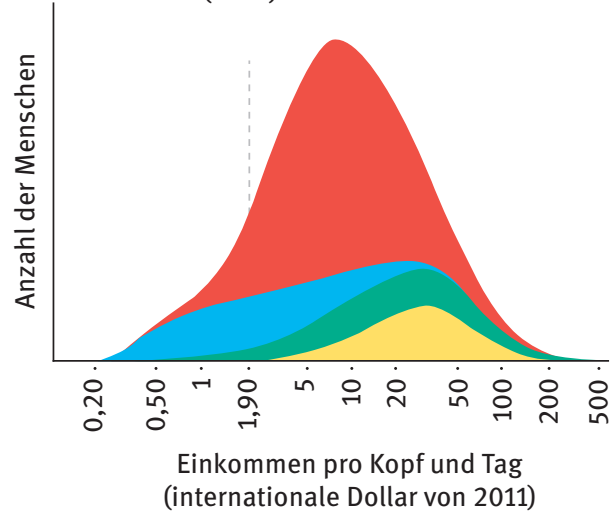

(b) 1930

2,1 Mrd. Menschen auf der Welt

1,3 Mrd. (62\%) leben in extremer Armut

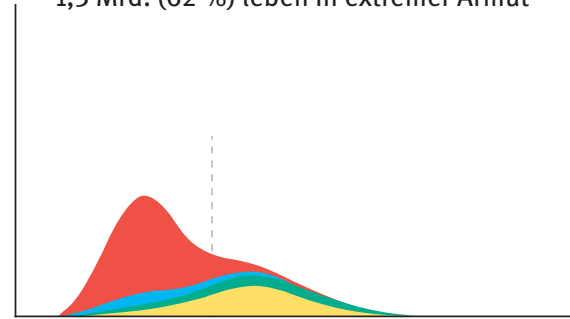

(d) 1980

4,4 Mrd. Menschen auf der Welt

2,1 Mrd. (48 \%) leben in extremer Armut

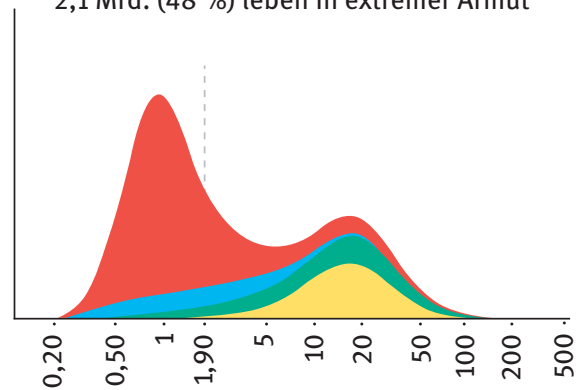

Einkommen pro Kopf und Tag

(internationale Dollar von 2011)

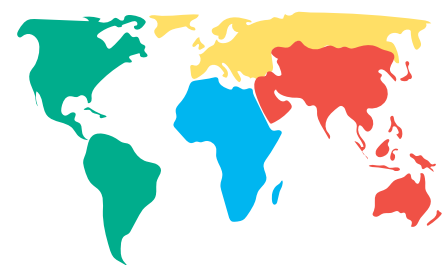

Abb. 19.2: Globale Einkommensverteilung von 1800 bis 2019. Einkommen entsprechen dem Bruttoinlandsprodukt pro Kopf und Tag. Deren inländische Verteilungen wurden aus Gini-Indexen abgeleitet. Einkommen > 500 internationale Dollar von 2011 sind nicht dargestellt. Die senkrechte Linie zeigt die internationale Armutsgrenze bei 1,90 internationale Dollar pro Tag. Die waagrechte Achse ist logarithmisch eingeteilt. Quelle: Modifizierte, frei zugängliche Abbildung von Gapminder.org, CC-BYLizenz. 


\section{Extreme Armut - unterhalb der internationalen Armutsgrenze}

Extreme Armut ist eine Form von absoluter Armut. Menschen, die in extremer Armut leben, haben unzureichenden Zugang zu erforderlicher Ernährung und anderen Artikel des täglichen Bedarfs. Die Weltbank verwendet seit 2015 eine internationale Armutsgrenze (International Poverty Line) von 1,90 internationalen Dollar von 2011 pro Tag. Einführt wurde diese internationale Armutsgrenze im Jahr 1990 mit etwa 1 internationaler Dollar von 1985 pro Tag.

Extreme Armut versucht die Zahl der bedürftigsten Menschen erfassen. Auch Lebensbedingungen weit über der internationalen Armutsgrenze können von Armut und Not geprägt sein, sodass neben der internationalen Armutsgrenze weitere Maße genutzt werden, wie beispielsweise die Lower (Upper) Middle Income Class Poverty Line bei 3,20 (5,50) internationalen Dollar von 2011 pro Tag oder der Human Development Index, um verschiedene Arten von Armut und deren Entwicklung zu messen.

Bei der Anwendung der internationalen Armutsgrenze zur Abschätzung der Anzahl der Menschen, die in extremer Armut leben, werden sowohl Veränderungen in den Bedürfnissen und Preisen über die Zeit als auch Unterschiede in der Kaufkraft zwischen Ländern berücksichtigt. Um abzuschätzen wie viele Menschen in extremer Armut leben unterstützt die Weltbank bei aktuellen Daten die Durchführung von Haushaltsbefragungen, um konsumierte Güter und Dienstleistungen und deren Wert pro Kopf zu ermitteln. Dadurch bezieht Armut auch den Verzehr oder Verbrauch von selbst hergestellten Gütern und Dienstleistungen ein, von denen Menschen leben, die für den eigenen Verbrauch benötigte Güter nicht einkaufen, sondern selbst produzieren (Subsistenzwirtschaft). In Abb. 19.2 wurden die globale Einkommensverteilung und der Anteil der Menschen in extremer Armut auf Grundlage von Bruttoinlandsprodukt pro Kopf-Werten, Bevölkerungszahlen und Informationen über die Ungleichheit innerhalb von Ländern abgeschätzt.

\subsubsection{Abnahme der globalen Einkommensungleichheit seit Ende des 20. Jahrhunderts}

Nach einer stetigen Zunahme der globalen Ungleichheit während des 19. Jahrhundert, bis in die 1980er oder noch 1990er Jahre [18], hat sich die weltweite Einkommensverteilung, vor allem durch wirtschaftliches Wachstum in China und Indien, erneut stark verändert. Die weltweite Einkommensverteilung hat sich nach rechts verschoben, das heißt, das globale Medianeinkommen, also das Einkommen, bei dem es genauso viele Menschen mit einem höheren wie mit einem niedrigeren Einkommen gibt, ist angestiegen und die Mehrheit der Welt ist wohlhabender geworden [19]. Da Einkommen des ärmeren Teils der Weltbevölkerung mit einer höheren Rate als die Einkommen des reicheren Teils der Weltbevölkerung wuchsen, ist die globale Einkommensungleichheit möglicherweise bereits seit den 1980er Jahren und besonders seit den 2000 Jahren zurückgegangen [18]. Dabei hat sich die zweigipflige Einkommensverteilung seit den späten 1990er Jahren wieder in eine eingipflige Verteilung umgewandelt, und die Weltregion, in der die meisten Menschen in Armut leben, hat sich von Asien zu Afrika geändert (Abb. 19.2e).

Obwohl durch das Wachstum niedriger Einkommen die globale Ungleichheit abgenommen hat und die deutliche Zweiteilung der Welt nach Einkommen verschwun- 
den ist, besteht weiterhin eine hohe globale Einkommensspreizung. Der Großteil der Weltbevölkerung lebt von sehr niedrigen Einkommen und nur wenige Menschen erzielen höhere Einkommen. Dies verdeutlicht Abb. 19.3, welche im Gegensatz zu Abb. 19.2 die Einkommensverteilung nicht auf einer logarithmischen Achse für Einkommen pro Tag, sondern auf einer linearen Achse für Jahreseinkommen bis 14.000 internationale Dollar von 2011 (etwa 38 internationale Dollar pro Tag) darstellt. Das Medianeinkommen hat sich innerhalb der Dekade von 2003 bis 2013 nahezu verdoppelt. Zugleich lag das Einkommen der wohlhabendsten $10 \%$ der Bevölkerung im Jahr 2013 bei 14.500 internationale Dollar oder höher, während das Einkommen der ärmsten 10 \% der Bevölkerung 480 internationale Dollar von 2011 oder weniger betrug - das Verhältnis dieser beiden Werte ist $30 \mathrm{zu} 1$.

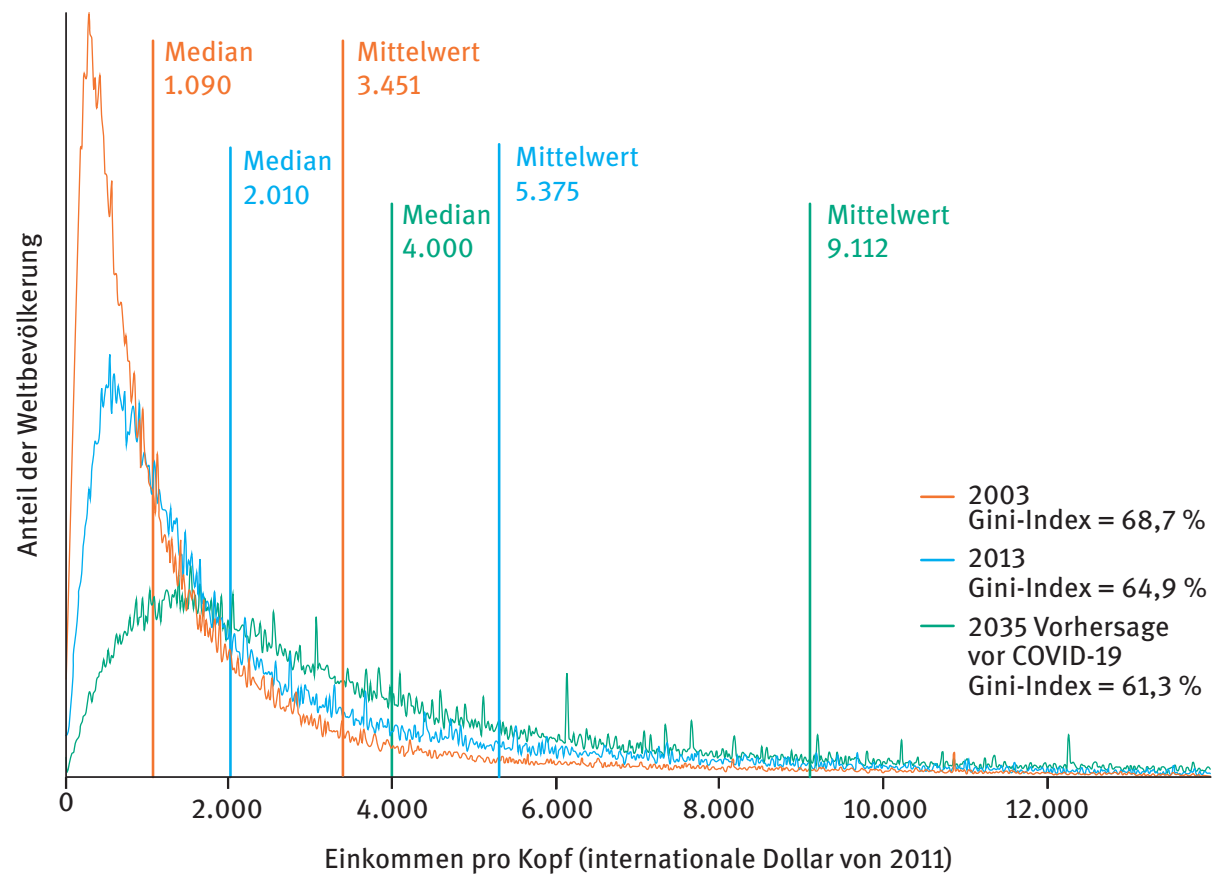

Abb. 19.3: Globale Einkommensverteilung in den Jahren 2003, 2013 und 2035. Einkommen entsprechen dem mittleren Bruttoinlandsprodukt pro Kopf und Jahr. Einkommen > 14.000 internationale Dollar von 2011 sind nicht dargestellt. Die jährlichen Wachstumsraten des Bruttoinlandsprodukts pro Kopf von 2014 bis 2035, welche der Prognose der globalen Einkommensverteilung für 2035 zugrunde liegen sind 2,0 \% für die Europäische Union, 2,2 \% für andere Mitgliedsländer der Organisation für wirtschaftliche Zusammenarbeit und Entwicklung, 1,9 \% für Nordamerika, 3,1 \% für Osteuropa und Zentralasien, 2,2 \% für Lateinamerika und Karibik, 1,8 \% für den Mittleren Osten und Nordafrika, 3,2 \% für Südasien, 4,4 \% für China, 4,5 \% für Ostasien und den Pazifik, 4,8 \% für Indien und 3,5\% für Subsahara-Afrika. Quelle: In Anlehnung an Hellebrandt und Mauro 2015 [20]. 


\subsubsection{Ausblick auf die globale Einkommensverteilung im Jahr 2035}

Ein häufig genutztes Maß zur Beschreibung der Ungleichheit einer Verteilung ist der Gini-Index. Der Gini-Index wurde vom italienischen Statistiker Corrado Gini entwickelt und im Jahr 1912 veröffentlicht. Bei einer auf Werte zwischen von 0 bis $100 \%$ normierten Skala ergibt sich der Wert $0 \%$ bei einer Gleichverteilung (z. B. von Einkommen) und der Wert $100 \%$ bei maximaler Ungleichverteilung (z. B. ein Individuum erhält das gesamte Einkommen, alle anderen erhalten keine Einkommen). Unter Annahme der Wirtschaftswachstumsraten, die unter Abb. 19.3 schrieben sind, würde die globale Ungleichheit in der Einkommensverteilung weiter abnehmen. Bis zum Jahr 2035 könnte der Gini-Index auf 61,3 \% absinken, im Vergleich zu 64,9 \% im Jahr 2013 und 68,7 \% im Jahr 2003, und das Medianeinkommen auf 4.000 internationale Dollar von 2011 ansteigen.

Die durchschnittliche Wachstumsrate des Pro-Kopf-Bruttoinlandsprodukt zwischen 2014 und 2019 betrug in Ländern mit hohem Einkommen 1,6 \%. In Länder mit oberem mittlerem Einkommen, Länder mit niedrigem mittlerem Einkommen und Länder mit niedrigem Einkommen wuchs die Wirtschaft im Durchschnitt der jeweiligen Ländergruppe um 3,4 \%, 3,7 \% und 0,9 \% (Abb. 19.4f). Bei einer Wachstumsrate von $2 \%$ dauert es 35 Jahre bis sich ein Einkommen verdoppelt, bei $5 \%$ dauert es 14 Jahre, bei $10 \%$ dauert es 7 Jahre. Trotz Anhaltspunkten für eine im Trend der letzten beiden Jahrzehnte bis zur COVID-19-Pandemie sinkende Ungleichheit in der weltweiten Einkommensverteilung wird es deshalb voraussichtlich lange dauern bis die niedrigsten Einkommen auf das heutige Niveau der höheren Einkommen ansteigen $[19,20]$. Im Hinblick auf die bestehenden gesundheitlichen Unterschiede in der Welt legen die Preston Kurve und medizinisch-technischer Fortschritt nahe, dass gesundheitliche Verbesserungen teilweise auch bei geringer oder ohne Wohlstandszunahme möglich sind und somit einer Abnahme globaler Einkommensunterschiede vorausgehen können (Abb. 19.1).

\subsubsection{Erneute Zunahme der globalen Einkommensungleichheit durch die COVID-19-Pandemie}

Bis zur COVID-19-Pandemie wurde erwartet, dass im Jahr 2020 noch etwa $10 \%$ der Weltbevölkerung in extremer Armut leben. Die sich nach wie vor entwickelnde Pandemie könnte den 20-jährigen Trend abnehmender extremer Armut in der Welt umkehren. Nach Schätzungen werden in den Jahren 2020 und 2021 zwischen 110 und 150 Millionen Menschen neu in extreme Armut gedrängt. Dies entspricht einem $\mathrm{Zu}$ rückfallen um 5 oder mehr Jahre bei der Anzahl der auf der Welt in extremer Armut lebenden Menschen. Der wirtschaftliche Schaden der Pandemie wird voraussichtlich Menschen aller Einkommensklassen in nahezu allen Ländern betreffen, aber Menschen mit niedrigen Einkommen überdurchschnittlich hart. Ohne starke Gegenmaß- 
nahmen wird durch die COVID-19-Pandemie nicht nur das globale Einkommen sinken und die extreme Armut zunehmen, sondern auch die weltweite Einkommensungleichheit seit langem erstmalig wieder zunehmen. Andere Pandemien, wie SARS im Jahr 2003 und Zika im Jahr 2016, haben Einkommen und Einkommensverteilung weltweit vermutlich ähnlich, aber in geringerem Maße beeinflusst [21].

\subsubsection{Innerstaatliche und zwischenstaatliche Einkommensungleichheit}

Anfang des 19. Jahrhunderts war die globale Einkommensungleichheit größtenteils auf Einkommensunterschiede innerhalb von Ländern zurückzuführen. Seit den 1930er Jahren ist die Ungleichheit der Einkommen zwischen verschiedenen Ländern höher als die Ungleichheit der Einkommen innerhalb von Ländern. Die innerstaatliche Einkommensungleichheit bewegte sich über viele Jahrzehnte auf ähnlichem Niveau. Sie sank zwischen dem Ende des Ersten Weltkriegs und der Nachkriegszeit des Zweiten Weltkriegs deutlich und nimmt im Gesamten vermutlich seit den 1990er Jahren wieder zu. Im Gegensatz dazu nahm die Einkommensungleichheit zwischen Staaten, welche über fast zwei Jahrhunderte stetig zugenommen hatte, zwischen den 1990er Jahren und dem Beginn der COVID-19-Pandemie im Jahr 2020 ab. Da die Ungleichheit in der Einkommensverteilung zwischen Ländern abnahm, ging die globale Einkommensungleichheit bis 2020 zurück, obwohl innerhalb mancher Länder die Einkommensungleichheit zunahm [18-22].

Die aufstrebenden Volkswirtschaften China und Indien verzeichneten jahrelang hohe Wachstumsraten, die wesentlich zum Rückgang der globalen Armut beitrugen. Gleichzeitig wurde die Einkommensspreizung zwischen verschiedenen Bevölkerungsschichten innerhalb dieser, wie auch anderer Staaten größer, da Wohlstandsgewinne nicht allen Menschen im Land in gleichem Maße zugutekamen. Aktuelle Daten deuten zumindest für einige Länder, in denen die innerstaatliche Einkommensungleichheit in den letzten Jahrzehnten zugenommen hat, auf eine Trendwende hin. In China scheint die innerstaatliche Ungleichheit, nach Erreichen eines Plateaus, seit Mitte der ersten Dekade des 20. Jahrhunderts abzunehmen [23]. Auch in zahlreichen Ländern Lateinamerikas erscheint die wirtschaftliche Ungleichheit seit dem Jahr 2000 wieder rückläufig [24]. Zunehmende innerstaatliche Ungleichheit wird indessen seit einigen Jahren vermehrt aus Ländern mit hohem Einkommensniveau berichtet $[25,26]$.

Eine genaue Abschätzung von Ungleichheit in einem Land oder zwischen Ländern ist allerdings aufgrund knapper Daten und teils schwieriger Bewertbarkeit von Werten (z. B. eines Sozialsystems) nicht nur für die Vergangenheit, sondern auch die Gegenwart eine Herausforderung (s. [25]). 


\subsubsection{Einkommensungleichheit und Gesundheit}

Ob und wie sich Einkommensungleichheit auf die Gesundheit der Bevölkerung auswirkt ist eine wichtige Frage, insbesondere bei vorhandener oder ansteigender innerstaatlicher Einkommensungleichheit. Eine Vermutung ist, dass das höchste durchschnittliche Gesundheitsniveau in einem Land erzielt werden kann, wenn das verfügbare Einkommen in der Bevölkerung gleichverteilt ist, also alle Bürger gleich viel verdienen. Die Überlegung hinter dieser Vermutung ist, dass ärmere Menschen, unter sonst gleichen Umständen, mit zusätzlichem Einkommen mehr für ihre Gesundheit tun können als bereits wohlhabendere Menschen. Es wird also, ähnlich wie bei der Preston Kurve im Ländervergleich (Abb. 19.1), beim Einkommen des einzelnen Menschen eine abnehmende Nützlichkeit im Hinblick auf die Möglichkeit, damit eine Verbesserung der eigenen Gesundheit zu erwirken, vermutet.

Falls dies der Fall ist und ärmere Menschen durch zusätzliches Einkommen ihre Gesundheit mehr verbessern können als wohlhabendere Menschen, dann würde durch eine Verringerung der einer Einkommensungleichheit in einer Bevölkerung deren durchschnittliche Gesundheit steigen. Jedoch hat in dieser Überlegung nicht die Einkommensungleichheit selbst eine Auswirkung auf die Gesundheit, sondern der unterschiedliche für die Gesundheit nutzbare Wert von Einkommen bei ungleicher Einkommensverteilung. Dies bedeutet der Mehrwert von zusätzlichem Einkommen für die Gesundheit hängt ursächlich von der Höhe des Einkommens und nicht vom Unterschied zu anderen Einkommen ab. Die Folge ist, Verbesserungen der Gesundheit würden durch höhere Einkommen und nicht geringere Ungleichheit für sich alleingenommen erzielt. Es könnte also eine Scheinkorrelation (Korrelation ohne Kausalzusammenhang) vorliegen, wenn ein Zusammenhang zwischen Einkommensungleichheit und Gesundheit beobachtet wird.

Auswirkungen von Einkommensungleichheit auf die Gesundheit (Kausalzusammenhänge) werden allerdings auch aus anderen Gründen vermutet [27,28]. Menschen in ungleichen Gesellschaften vertrauen ihren Mitmenschen weniger. Geringeres Vertrauen kann soziale Verbindungen und Netzwerke, welche die Gesundheit fördern, schwächen. Menschen, die empfinden, unfair entlohnt zu werden, zeigen mit höherer Wahrscheinlichkeit körperliche Stress-Symptome [29]. Neben der Wahrnehmung von Ungleichheit mit möglichen gesundheitlichen Folgen werden auch Zusammenhänge zwischen der Einkommensungleichheit und Schulbildung, Gesundheitsvorsorge, Arbeitsbedingungen oder Kriminalitätsraten vermutet, welche sich im Laufe des Lebens auf die Gesundheit auswirken.

Die Aussagekraft und Auslegung zahlreicher Studien zu Einkommensungleichheit und Gesundheit sind umstritten, da ein Scheinzusammenhang, der eigentlich auf anderen Faktoren beruht, nicht hinreichend ausgeschlossen wurde oder auch Erkrankungen Einkommensungleichheiten auslösen oder verstärken können (umgekehrter Kausalzusammenhang). Eine Übersichtsarbeit hat Literatur zu der Frage, wie sich innerstaatliche Einkommensungleichheit auf die Gesundheit auswirkt, systema- 
tisch zusammengetragen und konkurrierende Erklärungsansätze (vergleichbar mit den Erklärungen im Kasten in Kap. 19.2.4) untersucht. Deren Schlussfolgerung ist, dass insbesondere aufgrund der Ergebnisse neuerer Studien ein Kausalzusammenhang wahrscheinlich ist und somit in den meisten Fällen Einkommensungleichheit schlecht für die Gesundheit der Bevölkerung sei [30].

\subsection{Gesundheitsausgaben und Finanzierung im internationalen Vergleich}

\subsubsection{Wohlstand und Gesundheitsausgaben}

Weltweit wurden im Jahr 2019 schätzungsweise mehr als 10 Billionen internationale Dollar zu 2019 Preisen für Gesundheit ausgegeben. Etwa zwei Drittel der weltweiten Ausgaben für Gesundheit 2019 wurden in Länder mit hohem Einkommen ausgegeben, in welchen etwa ein Sechstel der Weltbevölkerung leben. Weniger als ein Prozent der weltweiten Gesundheitsausgaben wurden in Länder mit niedrigem Einkommen ausgegeben, in denen knapp ein Zehntel der Weltbevölkerung lebt. In Ländern mit hohem Einkommen haben Gesundheitsausgaben durchschnittlich einen mehr als zweimal so großen Anteil am Bruttoinlandsprodukt als in den Ländergruppen mit niedrigeren Einkommensniveaus (Tab. 19.1).

Unter Berücksichtigung der Kaufkraftunterschiede verschiedener nationaler Währungen, durch Umrechnung in internationale Dollar von 2019, ergeben sich ProKopf-Ausgaben von 6.168 internationalen Dollar in Ländern mit hohem Einkommen im Vergleich zu 1.148 internationalen Dollar in Ländern mit oberem mittlerem Einkommen, 312 internationalen Dollar in Ländern mit niedrigem mittlerem Einkommen und 121 internationalen Dollar in Ländern mit niedrigem Einkommen. Die drei Länder mit den höchsten Pro-Kopf-Gesundheitsausgaben im Jahr 2019 waren, unter Berücksichtigung von Kaufkraftunterschieden, die USA (10.976 internationale Dollar von 2019, 16,9 \% des Bruttoinlandsprodukts), Andorra (9.940 internationale Dollar, $8 \%$ ) und Norwegen (8.120 internationale Dollar, 10,6\%). Die geringsten Gesundheitsausgaben pro Kopf wurden für Somalia (13 internationale Dollar, 4,6 \% des Bruttoinlandsprodukts), die Demokratische Republik Kongo (30 internationale Dollar, 3,5 \%) und die Zentralafrikanischen Republik (36 internationale Dollar, 4,5 \%) bzw. Eritrea (36 internationale Dollar, 5,3 \%) berichtet. In Deutschland betrugen die Gesundheitsausgaben im Jahr 2019 schätzungsweise 6.124 internationale Dollar von 2019 pro Kopf (11,4 \% des Bruttoinlandsprodukts). 
Tab. 19.1: Bevölkerung, Lebenserwartung, Krankheitslast und Gesundheitsausgaben nach Ländereinkommensgruppe im Jahr 2019, in Prozent des globalen Werts und prozentuale Veränderung seit 1995.

\begin{tabular}{|c|c|c|c|c|c|c|c|}
\hline \multirow{3}{*}{$\begin{array}{l}\text { Länder- } \\
\text { einkommens- } \\
\text { gruppe }\end{array}$} & \multirow{3}{*}{$\begin{array}{l}\begin{array}{l}\text { Bevöl- } \\
\text { kerung }\end{array} \\
\\
\text { Mrd. } \\
\% \text { Welt } \\
\% \uparrow \downarrow\end{array}$} & \multirow{3}{*}{$\begin{array}{l}\text { Lebenser- } \\
\text { wartung } \\
\text { bei } \\
\text { Geburt } \\
\\
\text { Jahre } \\
\% \text { Welt- } \varnothing \\
\% \uparrow \downarrow\end{array}$} & \multirow{2}{*}{\multicolumn{2}{|c|}{$\begin{array}{l}\text { Krankheitslast } \\
\text { Disability-Adjusted Life } \\
\text { Years }\end{array}$}} & \multirow{2}{*}{\multicolumn{2}{|c|}{$\begin{array}{l}\begin{array}{l}\text { Gesundheits- } \\
\text { ausgaben }\end{array} \\
\text { Internationale } \\
\text { Dollar von } 2019\end{array}$}} & \multirow[b]{3}{*}{$\begin{array}{l}\text { \% Brutto- } \\
\text { inlandsproduk } \\
\% \text { Welt- } \varnothing \\
\% \uparrow \downarrow\end{array}$} \\
\hline & & & & & & & \\
\hline & & & 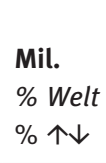 & $\begin{array}{l}\text { pro } 100000 \\
\text { Menschen } \\
\% \text { Welt- } \varnothing \\
\% \uparrow \downarrow\end{array}$ & $\begin{array}{l}\text { Mrd. } \\
\% \text { Welt } \\
\% \uparrow \downarrow\end{array}$ & $\begin{array}{l}\text { pro Kopf } \\
\% \text { Welt- } \varnothing \\
\% \uparrow \downarrow\end{array}$ & \\
\hline Welt & $\begin{array}{l}7,7 \\
100 \\
34,5\end{array}$ & $\begin{array}{l}72,7 \\
100 \\
9,7\end{array}$ & $\begin{array}{l}2538 \\
100 \\
-3,4\end{array}$ & $\begin{array}{l}32802 \\
100 \\
-28,2\end{array}$ & $\begin{array}{l}11.594 \\
100 \\
166\end{array}$ & $\begin{array}{l}1.499 \\
100 \\
98,9\end{array}$ & $\begin{array}{l}9,9 \\
100 \\
22\end{array}$ \\
\hline $\begin{array}{l}\text { Länder mit } \\
\text { hohem } \\
\text { Einkommen }\end{array}$ & $\begin{array}{l}1,2 \\
16,1 \\
15,8\end{array}$ & $\begin{array}{l}80,8 \\
111 \\
6,2\end{array}$ & $\begin{array}{l}357 \\
14,1 \\
13,59\end{array}$ & $\begin{array}{l}29758 \\
90,7 \\
-2,4\end{array}$ & $\begin{array}{l}7.446 \\
64,2 \\
132\end{array}$ & $\begin{array}{l}6.168 \\
412 \\
99,3\end{array}$ & $\begin{array}{l}12,5 \\
126 \\
39\end{array}$ \\
\hline $\begin{array}{l}\text { Länder mit } \\
\text { oberem } \\
\text { mittlerem } \\
\text { Einkommen }\end{array}$ & $\begin{array}{l}2,9 \\
37,2 \\
22,6\end{array}$ & $\begin{array}{l}75,5 \\
104 \\
9,7\end{array}$ & $\begin{array}{l}752 \\
29,6 \\
-1,09\end{array}$ & $\begin{array}{l}28426 \\
86,7 \\
-16,7\end{array}$ & $\begin{array}{l}3.095 \\
26,7 \\
270\end{array}$ & $\begin{array}{l}1.148 \\
76,6 \\
209\end{array}$ & $\begin{array}{l}5,9 \\
59,6 \\
13\end{array}$ \\
\hline $\begin{array}{l}\text { Länder mit } \\
\text { niedrigem } \\
\text { mittlerem } \\
\text { Einkommen }\end{array}$ & $\begin{array}{l}2,9 \\
38,8 \\
48,6\end{array}$ & $\begin{array}{l}68,6 \\
94,4 \\
13,4\end{array}$ & $\begin{array}{l}1105 \\
43,5 \\
-6,2\end{array}$ & $\begin{array}{l}34733 \\
106 \\
-37,6\end{array}$ & $\begin{array}{l}966 \\
8,3 \\
273\end{array}$ & $\begin{array}{l}312 \\
20,8 \\
148\end{array}$ & $\begin{array}{l}3,9 \\
39,4 \\
5,4\end{array}$ \\
\hline $\begin{array}{l}\text { Länder mit } \\
\text { niedrigem } \\
\text { Einkommen }\end{array}$ & $\begin{array}{l}0,7 \\
8,7 \\
90,6\end{array}$ & $\begin{array}{l}63,8 \\
87,8 \\
23,3\end{array}$ & $\begin{array}{l}323 \\
12,7 \\
-13,9\end{array}$ & $\begin{array}{l}45653 \\
139 \\
-53,8\end{array}$ & $\begin{array}{l}89 \\
0,8 \\
101\end{array}$ & $\begin{array}{l}121 \\
8,1 \\
8,0\end{array}$ & $\begin{array}{l}5,2 \\
52,5 \\
4,0\end{array}$ \\
\hline
\end{tabular}

Lebenserwartungen und Gesundheitsausgaben für 2019 sind Vorhersagen. Welt- $\varnothing=$ Weltdurchschnitt. $\uparrow \downarrow=$ Veränderung seit 1995. Datenquellen: Financing Global Health Studie 2019, Global Burden of Disease Studie 2019 und World Development Indicators.

Die Gesundheitsausgaben eines Landes sind also, ähnlich wie die Gesundheit der Bevölkerung eines Landes, eng mit dem Bruttoinlandsprodukt verbunden. Je größer das Bruttoinlandsprodukt pro Kopf, desto größer sind im Durchschnitt die Ausgaben für die Gesundheit insgesamt und pro Kopf. In Ländern mit niedrigem Einkommen sind allerdings die durchschnittlichen Gesundheitsausgaben im Verhältnis zum Bruttoinlandsprodukt höher als in Ländern mit niedrigem mittlerem Einkommen. Dies liegt zum einen am niedrigeren Pro-Kopf-Bruttoinlandsprodukt der Länder mit niedrigem Einkommen und zum anderen an der höheren Unterstützung, die diese Länder von Gebern durch Entwicklungshilfe für Gesundheit (Development Assistance for Health) erhalten. 
Der deutlich höhere Anteil der Gesundheitsausgaben am Bruttoinlandsprodukt der Länder mit hohem Einkommen beinhaltet einerseits die Versorgung einer alternden Bevölkerung, deren vorrangige Erkrankungen chronisch sind und laufende Gesundheitsausgaben zur Folge haben. Andererseits können die anteilig höheren Gesundheitsausgaben der Länder mit hohem Einkommen einen zunehmenden Stellenwert von zusätzlichen Ausgaben für Gesundheit widerspiegeln, nachdem für grundlegende Bedürfnisse gesorgt ist.

Menschen, die in Armut und ohne Krankenversicherung leben, indessen haben oft in verschiedenen Lebensbereichen untererfüllte Grundbedürfnisse und nicht versicherte Risiken. Eine kostenverursachende und Arbeitszeit in Anspruch nehmende Behandlung einer Erkrankung steht für Menschen in Armut deshalb eher im Konflikt mit anderen Bedürfnissen. Dies gilt insbesondere in einem frühen oder nicht-akuten Stadium einer Erkrankung und für Gesundheitsvorsorge. Mit der Fürsorge und Ausgaben für die eigene Gesundheit konkurrieren Notwendigkeiten, wie Geld zu sparen oder Einkommen zu erzielen und dieses für andere Zwecke auszugeben (z. B. um eine Familie zu ernähren [31], eine Ausbildung der Kinder zu finanzieren, einen belastenden Kredit mit hohem Zinssatz zurückzuzahlen oder einem Familienmitglied zu helfen, dem es akut schlechter geht).

Da Armut mit vielen unmittelbaren Nöten verbunden sein kann, nehmen mit zunehmendem Wohlstand die Möglichkeiten zu, für die Zukunft und über das Notwendigste hinaus vorzusorgen. Das bedeutet, es steigt der relative Stellenwert der Gesundheit mit dem Wohlstandsniveau und in Folge auch der Anteil des verfügbaren Einkommens, der für Gesundheitsausgaben aufgewendet werden kann. Für Länder mit hohem Einkommen wurde geschätzt, dass das sozial wünschenswerte Niveau der Gesundheitsausgaben bei über $30 \%$ des Bruttoinlandsprodukts liegen könnte [32]. Im Jahr 2019 wendeten Länder mit hohem Einkommen im Durschnitt schätzungsweise 12,5\% des Bruttoinlandsprodukts für Gesundheitsausgaben auf.

\subsubsection{Gesundheitsausgaben und Krankheitslast}

Im globalen Durchschnitt betrug die geschätzte Krankheitslast im Jahr 2019 gemessen in behinderungsbereinigten Lebensjahren (Disability-Adjusted Life Years, DALYs) 2,5 Milliarden DALYs oder 32802 DALYs pro 100000 Menschen (Tab. 19.1). Das bedeutet, dass weltweit fast jedes dritte Lebensjahr, das vermutlich gesund gelebt werden könnte, durch vorzeitigen Tod oder krankheitsbedingte Beeinträchtigungen eingebüßt wird. Im Mittel nimmt die Schwere der Krankheitslast mit zunehmendem Wohlstand ab. Länder mit oberem mittlerem Einkommen und Länder mit hohem Einkommen haben bereits eine ähnliche Krankheitslast pro 100000 Einwohner. Mit 45653 DALYs pro 100000 Menschen ist die durchschnittliche Krankheitslast der Bevölkerung in Ländern mit niedrigem Einkommen etwa 50 \% schwerer als die Krankheitslast der Bevölkerungen in Ländern mit hohem oder oberem mittlerem Einkom- 
men. Die meiste Krankheitslast tritt mit 1,9 Mrd. DALYs in den Ländern mit mittlerem Einkommen auf, in denen $76 \%$ der Weltbevölkerung lebt.

Vergleicht man die aktuellen Werte der weltweiten Gesundheitsausgaben und der Verteilung der Krankheitslast, dann werden in Länder mit hohem Einkommen $64 \%$ der weltweiten Gesundheitsausgaben für $14 \%$ der weltweiten Krankheitslast ausgegeben. In Länder mit oberem mittlerem Einkommen treten $27 \%$ der globalen Gesundheitsausgaben und 30 \% der globalen Krankheitslast auf. Mit abnehmendem Wohlstand nimmt die Lücke zwischen Krankheitslast und Gesundheitsausgaben weiter zu. Länder mit niedrigem mittlerem Einkommen, in denen 8,3 \% der globalen Gesundheitsausgaben ausgegeben werden, haben mit $44 \%$ den größten Anteil der weltweiten Krankheitslast. Länder mit niedrigem Einkommen, in denen derzeit 8,7 \% der Weltbevölkerung leben, haben mit $13 \%$ den kleinsten Anteil an der globalen Krankheitslast, aber die höchste Krankheitslast pro 100000 Menschen und nur $0,8 \%$ der globalen Gesundheitsausgaben.

Trotz externer Unterstützung durch Entwicklungshilfe für Gesundheit, welche den Anteil der Gesundheitsausgaben am Bruttoinlandsprodukt anhebt, haben Länder mit niedrigem Einkommen das ungünstigste Verhältnis von Krankheitslast und Gesundheitsausgaben pro Kopf. Unter Berücksichtigung von Kaufkraftunterschieden werden in Ländern mit niedrigem mittlerem Einkommen etwa die 3,2-fachen, in Ländern mit oberem mittlerem Einkommen die 15-fachen und in Ländern mit hohem Einkommen die 76-fachen Gesundheitsausgaben pro DALY im Vergleich zu Ländern mit niedrigem Einkommen getätigt.

\subsubsection{Entwicklung von Bevölkerung, Gesundheit, Wohlstand und Gesundheitsausgaben von 1995 bis 2019}

In allen vier Ländereinkommensgruppen haben, im Durchschnitt, die Bevölkerungszahl, der Gesundheitszustand sowie die absolut und Pro-Kopf-Werte des Bruttoinlandsprodukts und der Gesundheitsausgaben in den zweieinhalb Jahrzehnten von 1995 bis 2019 zugenommen. Eine Verbesserung der durchschnittlichen Gesundheit wird für die meisten Ländereinkommensgruppen, in unterschiedlichem Ausmaß, durch einen Anstieg der Lebenserwartung bei Geburt und ein Absinken der Krankheitslast angezeigt. In Ländern mit hohem Einkommen stieg die Lebenserwartung wie in den andern Ländereinkommensgruppen durchgängig, doch nimmt dort die Krankheitslast pro 100000 seit dem Jahr 2010 altersbedingt zu (Abb. 19.4a-c).

Das stärkste wirtschaftliche Wachstum, insgesamt und pro Kopf, verzeichneten seit der Jahrtausendwende die Länder mit mittlerem oder niedrigem Einkommen. Das Bruttoinlandsprodukt pro Kopf ist seit der Jahrtausendwende am stärksten in Ländern mit oberem mittlerem Einkommen gewachsen, gefolgt Ländern mit niedrigem mittlerem Einkommen, dann Ländern mit niedrigem Einkommen und danach Ländern mit hohem Einkommen. Das stärkere Wachstum der Bevölkerung in Län- 
(a) Bevölkerung (Mrd. Menschen)

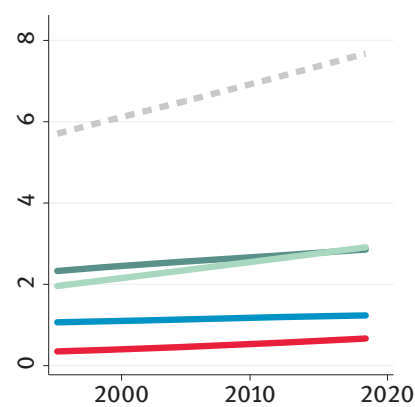

(d) Bruttoinlandsprodukt (Bil. internationale Dollar von 2017)

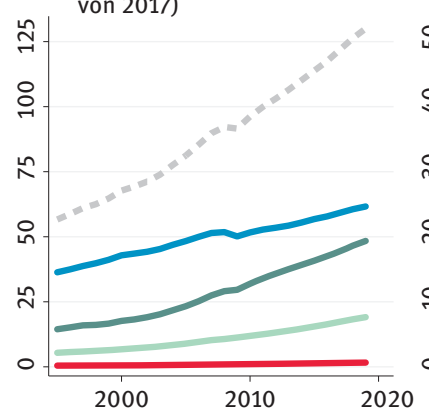

(g) Gesundheitsausgaben (Bil. internationale Dollar von 2019)

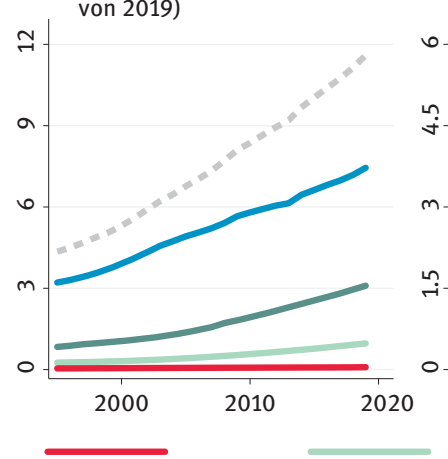

Länder mit niedrigem Einkommen (b) Lebenserwartung bei Geburt (Jahre)

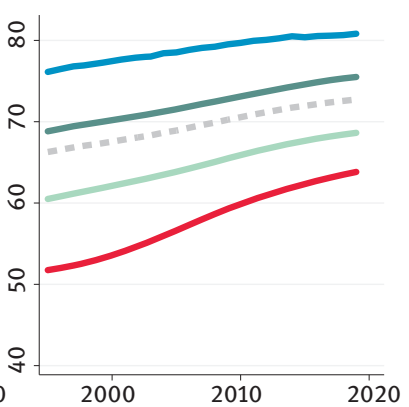

(e) Bruttoinlandsprodukt pro Kopf (Tsd. internationale Dollar von 2017)

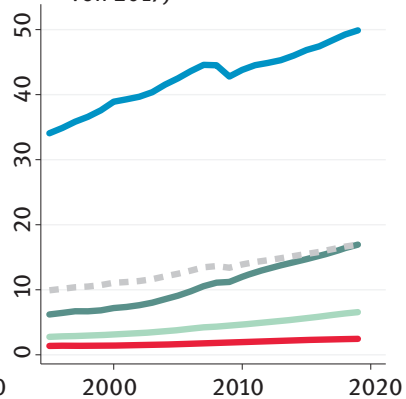

(h) Gesundheitsausgaben pro Kopf (Tsd. internationale Dollar von 2019)

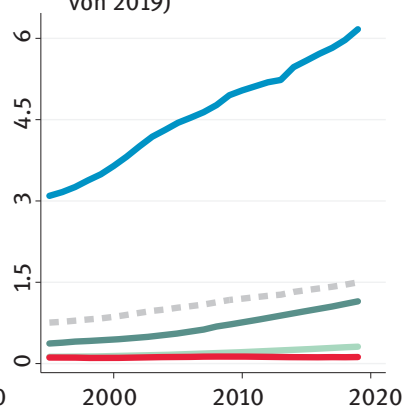

Länder mit niedrigem mittlerem Einkommen mittlerem Einkommen
Länder mit oberem (c) Krankheitslast (DALYs pro 100000 Menschen)

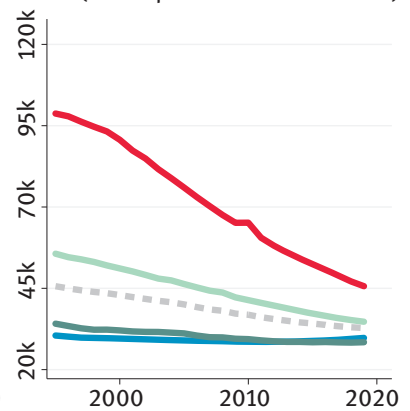

(f) Veränderung des Bruttoinlandsprodukts pro Kopf (\% gegenüber Vorjahr)

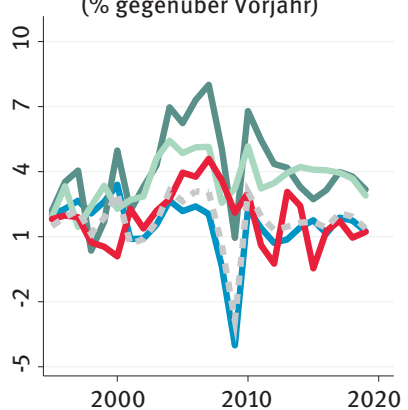

(i) Gesundheitsausgaben (\% des Bruttoinlandsprodukts von 2019)

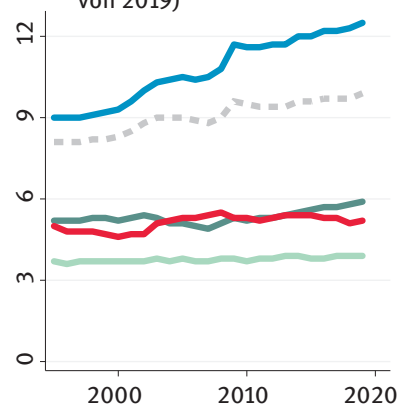

Länder mit

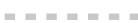

Welt

Abb. 19.4: Gesundheit, Wohlstand und Gesundheitsausgaben von 1995 bis 2019 nach Ländereinkommensgruppe. Unterschiede zwischen Ländern und im zeitlichen Verlauf sind durch Veränderungen der Bevölkerungsstruktur mitbedingt. Lebenserwartungen 2019 und Gesundheitsausgaben nach 2017 sind Vorhersagen. DALYs = Disability-Adjusted Life Years. Datenquellen: Financing Global Health Studie 2019, Global Burden of Disease Studie 2019 und World Development Indicators. 
dern mit niedrigem Einkommen im Vergleich zu den anderen Ländereinkommensgruppen bedeutet allerdings, dass ein stärkeres Wachstum des Bruttoinlandsprodukts als in anderen Ländereinkommensgruppen nötig ist, um ein vergleichbares Pro-Kopf-Wachstum zu erreichen (Abb. 19.4d-f).

Die größten Anstiege der Gesundheitsausgaben, insgesamt und pro Kopf, hatten Länder mit mittlerem Einkommen. Die Gesundheitsausgaben wuchsen dort mehr als doppelt so stark als in den Ländern mit hohen oder niedrigen Einkommen. Ähnlich wie beim Wachstum des Bruttoinlandsproduktes verteilen sich wachsende Gesundheitsaufgaben in Ländern mit niedrigen Einkommen auf eine Bevölkerung, die schneller wächst als in den anderen Ländereinkommensgruppen. Das Wachstum der Pro-Kopf-Gesundheitsausgaben in Ländern mit niedrigen Einkommen war mit 8 \% im Zeitraum von 1995 bis 2019 gering, verglichen zu einer Verdoppelung oder Verdreifachung der Pro-Kopf-Gesundheitsausgaben in den anderen Ländereinkommensgruppen. Der Anteil des Bruttoinlandsprodukts, welcher für Gesundheit ausgegeben wurde, war in Ländern mit niedrigem Einkommen eng mit der externen Unterstützung der Gesundheitssysteme durch Entwicklungshilfe für Gesundheit verbunden (Abb. 19.4g-i).

\subsubsection{Finanzierung der Gesundheitsausgaben}

Die Zielsetzung nationaler Gesundheitssysteme und globaler Bemühungen um die Gesundheit ist es, vermeidbare Tode und Krankheiten zu verhindern. Die Gesundheitsfinanzierung hat dabei die zentralen Aufgaben, den verschiedenen Bereichen des Gesundheitssystems finanzielle Mittel zur Verfügung zu stellen, Menschen vor finanziellen Folgen von schlechter Gesundheit zu schützen sowie eine wirtschaftliche Mittelverwendung und gerechte Gesundheitsversorgung zu fördern (s. Kasten).

\footnotetext{
Aufgaben der Gesundheitsfinanzierung

- Ausreichende Bereitstellung finanzieller Mittel für das Gesundheitswesen

- Schutz der Menschen vor finanziellen Folgen von schlechter Gesundheit

- Nutzung von Ressourcen in bestmöglicher Weise

- Gewährleisten einer gerechten Gesundheitsversorgung

Quelle: In Anlehnung an World Health Organization 2014 [33].
}

Gesundheitsausgaben können nach Ausgabenträger bzw. nach der vom Ausgabenträger abhängigen Finanzierungsquelle unterschieden werden in (s. Kap. 18.2.1):

1. Öffentliche Gesundheitsausgaben - beinhalten Steuern und Pflichtversicherungsbeiträge

2. Vorausbezahlte private Gesundheitsausgaben - beinhalten private Krankenversicherungsbeiträge und Gesundheitsausgaben von Nichtregierungsorganisationen 
3. Selbstzahlungen privater Haushalte - Zahlungen für die Gesundheit aus der eigenen Tasche (Out-of-Pocket Zahlungen)

4. Entwicklungshilfe für Gesundheit

In Länder mit hohem Einkommen wird der geringste Teil der Gesundheitsausgaben aus eigener Tasche im Krankheitsfall bezahlt (14\% im Jahr 2019) und die Gesundheitsversorgung wird ohne externe Unterstützung finanziert. Gesundheitsausgaben in Ländern mit hohem Einkommen waren im Jahr 2019 zu 86 \% durch öffentliche sowie vorausbezahlte private Gesundheitsausgaben finanziert. Menschen in Ländern mit hohem Einkommen sind durch diese weitgehend von eigener Krankheit unabhängige Gesundheitsfinanzierung somit in einem hohen Maße gegen finanzielle Risiken von Krankheit versichert. Die stärkste Zunahme bei den aus eigenen Mitteln versicherten Gesundheitsausgaben zwischen 1995 und 2019 gab es den Ländern mit oberem mittlerem Einkommen, in denen der Anteil der öffentlichen und vorausbezahlten privaten Gesundheitsausgaben von 61 \% im Jahr 1995 auf 68,1 \% im Jahr 2019 gestiegen ist. Nahezu der gesamte verbleibende Teil der Gesundheitsausgaben wurde in Ländern mit oberem mittlerem Einkommen aus der eigenen Tasche bezahlt (31,8 \% im Jahr 2019; Abb. 19.5).

Länder mit niedrigem oder niedrigem mittlerem Einkommen finanzieren weniger als die Hälfte der Gesundheitsausgaben durch öffentliche und vorausbezahlte private Gesundheitsausgaben (33 \% bzw. 46 \% im Jahr 2019). Der Anteil der Entwicklungshilfe für Gesundheit an den Gesundheitsausgaben war in Ländern mit niedrigem Einkommen am höchsten. Nach Berücksichtigung der Beiträge von Entwicklungshilfe für Gesundheit waren in Ländern mit niedrigem Einkommen, im Jahresdurchschnitt 2019, schätzungsweise $42 \%$ der Gesundheitsausgaben nicht versichert und wurden aus der eigenen Tasche bezahlt. In Ländern mit niedrigem mittlerem Einkommen war der Anteil der Selbstzahlungen mit 55 \% im Jahr 2019 am höchsten.

Die Entwicklungshilfe für Gesundheit betrug im Jahr 2019 etwa 40 Mrd. US-Dollar und stieg am stärksten in den 2000er Jahren (von 12 Mrd. US-Dollar im Jahr 2000 auf 35 Mrd. US-Dollar im Jahr 2010). Während die Entwicklungshilfe für Gesundheit im Jahr 2019 etwa 0,2 \% der globalen Gesundheitsausgaben darstellte, war deren Beitrag zur Unterstützung der Gesundheitsfinanzierung in Ländern mit niedrigeren Einkommen und für bestimmte globalen Gesundheitsmaßnahmen erheblich. Beispielsweise waren $26 \%$ der Gesundheitsausgaben der Länder mit niedrigem Einkommen im Jahr 2019 oder 48 \% der HIV/AIDS Ausgaben im Jahr 2017 Entwicklungshilfe für Gesundheit [34]. Eine Vorhersage der weltweiten Gesundheitsausgaben bis 2050 schätzt, dass in den meisten Ländern der Anteil an Selbstzahlungen erheblich und der Bedarf an Entwicklungshilfe für Gesundheit bestehen bleibt [35]. 
(a) Länder mit hohem Einkommen

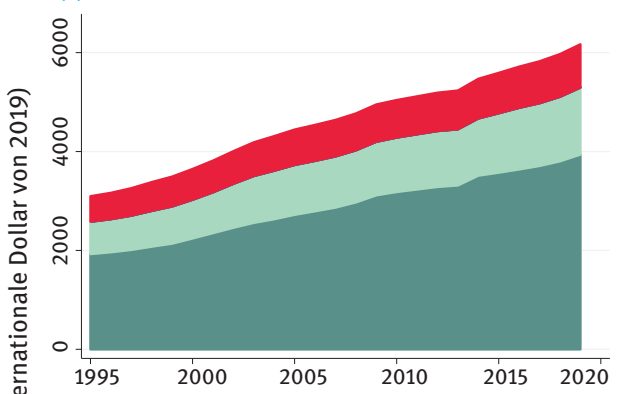

(c) Länder mit niedrigem mittlerem Einkommen

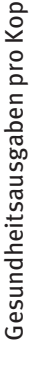

○-

1995

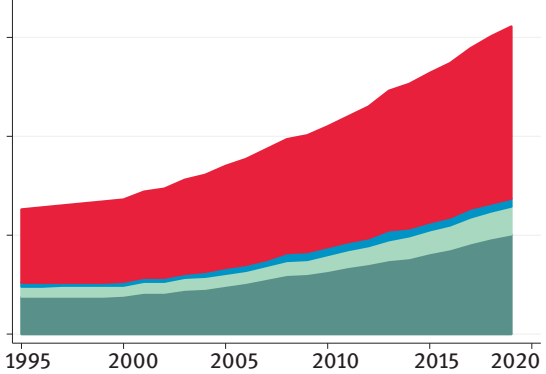

(b) Länder mit oberem mittlerem Einkommen

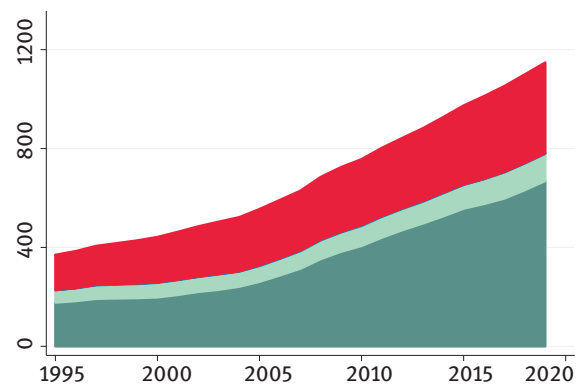

(d) Länder mit niedrigem Einkommen

욱

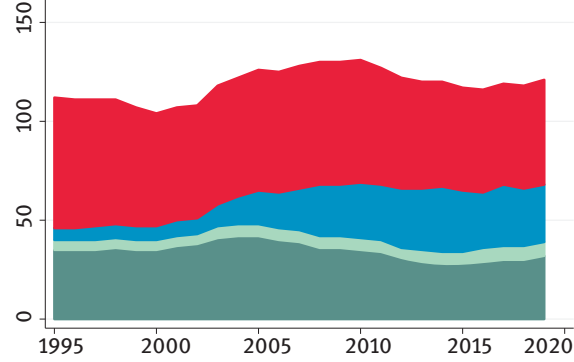

Öffentliche Gesundheitsausgaben

Entwicklungshilfe für Gesundheit

Vorausbezahlte private

Gesundheitsausgaben

Selbstzahlungen privater Haushalte für Gesundheitsleistungen

Abb. 19.5: Gesundheitsausgaben pro Kopf von 1995 bis 2019 nach Ausgabenträgern und Ländereinkommensgruppe. Öffentliche Gesundheitsausgaben beinhalten inländisch finanzierte öffentliche Ausgaben für Gesundheit. Vorausbezahlte private Gesundheitsausgaben schließen Privatversicherungen und Gesundheitsausgaben von Nichtregierungsorganisationen ein. Entwicklungshilfe für Gesundheit umfasst finanzielle Leistungen und Sachleistungen. Gesundheitsausgaben nach 2017 sind Vorhersagen. Datenquelle: Financing Global Health Studie 2019.

Neben dem Umfang der weltweit verfügbaren Entwicklungshilfe für Gesundheit bestimmt die Einkommensgruppe, der ein Land angehört, in welchem Ausmaß dessen Gesundheitsversorgung von Gebern im globalen Gesundheitsbereich unterstützt wird. Veränderungen im Wachstum und Fokus der Entwicklungshilfe für Gesundheit sowie ein Statusübergang von einem Land mit niedrigem Einkommen zu einem Land mit niedrigem mittlerem Einkommen können deshalb in ärmeren Ländern kritische Auswirkungen auf die Gesundheitsversorgung haben (s. Kasten und [34]). 
Ländereinkommensgruppen und Entwicklungshilfe für Gesundheit - die fehlende Mitte beim Wechsel der Gesundheitsfinanzierung

- Beim Wechsel der Gesundheitsfinanzierung (Health Financing Transition) findet ein Übergang weg von Selbstzahlungen und Entwicklungshilfe für Gesundheit hin zu einer Gesundheitsfinanzierung durch Steuern und Versicherungsbeiträge statt.

- Im Zuge des wirtschaftlichen Wachstums können Länder die „fehlende Mitte“ erleben, weil durch den Statusübergang von einem Land mit niedrigem Einkommen zu einem Land mit niedrigem mittlerem Einkommen die Entwicklungshilfe für Gesundheit meist sprunghaft abnimmt.

- Wenn die Finanzierung der Gesundheitsausgaben über Steuern und Versicherungsbeiträge noch nicht stark ausgeprägt ist, entsteht eine Finanzierungslücke, die durch Selbstzahlungen ausgeglichen werden muss.

Im Ergebnis bezahlen die Menschen in Ländern mit unterem mittlerem Einkommen, im Durchschnitt, den größten Anteil der Gesundheitsausgaben aus eigener Tasche. In Ländern mit oberem mittlerem Einkommen findet schließlich der Übergang weg von Selbstzahlungen hin zu einer überwiegenden Gesundheitsfinanzierung durch Steuern und Versicherungsbeiträge statt.

Wie die COVID-19-Pandemie die Finanzierung der Gesundheitsausgaben weltweit verändern wird, und wie Umfang und Zusammensetzung der Entwicklungshilfe für Gesundheit beeinflusst werden, sind offene Fragen. Die COVID-19-Pandemie betrifft die Finanzierung von Gesundheitsausgaben zum einen durch die akute Belastung der Gesundheitssysteme und zum anderen durch globale wirtschaftliche Folgen [34].

\subsubsection{Krankheit als Armutsfalle}

Eine Armutsfalle ist ein sich selbst verstärkender Mechanismus zwischen Armut und den Ursachen der Armut, der Menschen oder Länder dazu zwingt, arm zu bleiben, wenn keine Maßnahmen ergriffen werden, um den Teufelskreis zu durchbrechen.

Eigenleistungen und Zuzahlungen für die Behandlung von Krankheit können in allen Ländern der Welt dazu führen, dass Menschen als Folge der persönlichen Gesundheitsausgaben in die Armut gedrängt werden [36,37]. Nach Schätzungen geraten jedes Jahr 100 Millionen Menschen in Armut, weil sie im Krankheitsfall aus eigener Tasche für ihre Gesundheitsversorgung zahlen mussten [38]. Aus Sorge vor ruinösen Gesundheitsausgaben, die auch katastrophale Gesundheitsausgaben (Catastrophic Health Expenditures) genannt werden und einen so hohen Anteil des verfügbaren Einkommens eines Haushalts aufzehren, dass dieser Haushalt verarmt, vermeiden Menschen die Inanspruchnahme des Gesundheitssystems oder suchen dieses erst bei fortgeschrittenen Beschwerden auf. Eine späte Inanspruchnahme medizinischer Leistungen im Bedarfsfall kann wiederum das Risiko für Komplikationen und hohe Behandlungskosten erhöhen. 
Das Ausmaß wie sehr Menschen in Gefahr sind aus gesundheitlichen Gründen zu verarmen wird wesentlich von drei Faktoren bestimmt [39]:

1. dem Bedarf - Zugang zu und Nutzung von Gesundheitsleistungen

2. der Bedürftigkeit - eigene Zahlungsfähigkeit, Rücklagen und Vermögen

3. der Absicherung - Möglichkeiten zur Krankenversicherung

Da die größte und schwerste weltweite Krankheitslast in den Ländern mit niedrigem mittlerem und niedrigem Einkommen auftritt, in denen zudem große Teile der Gesundheitsausgaben aus einer Tasche bezahlt werden, sind besonders häufig Menschen, die dort leben, gleichzeitig einem höheren Erkrankungsrisiko und einem höheren krankheitsbedingten finanziellen Risiko ausgesetzt. Durch dieses gekoppelte Risiko steigt die Wahrscheinlichkeit, dass das Auftreten einer Erkrankung in eine Armutsfalle führt. Diese stellt dann eine Situation dar, in der durch krankheitsbedingten Verlust des Arbeitseinkommens die finanziellen Mittel für die Bezahlung einer notwendigen Behandlung fehlen und in der die bestehende Erkrankung die Wiederaufnahme der Arbeit verzögert oder völlig verhindert.

Eine ähnliche Abwärtsspirale kann ganze Volkswirtschaften betreffen. Fehlen in einem Land, aufgrund hoher Belastung durch Krankheiten, benötigte Arbeitskräfte, verschlechtert sich dessen Wirtschaftskraft und dadurch die Möglichkeiten des Landes Gesundheitsleistungen bereitzustellen. Verschlechtert sich in Folge einer unzureichenden Gesundheitsversorgung wiederum die gesundheitliche Lage auf der Bevölkerungsebene, dann können ganze Länder, durch ähnliche Mechanismen wie einzelne Familien, in eine Armutsfalle gelangen und darin gefangen bleiben. Im Gegenzug können medizinische Maßnahmen, die zu einer besseren Gesundheit der Bevölkerung eines Landes beitragen, zu einem wirtschaftlichen Aufschwung führen, welcher es schließlich erlaubt mehr benötigte und gewünschte Gesundheitsleistungen $\mathrm{zu}$ finanzieren (Abb. 19.6).

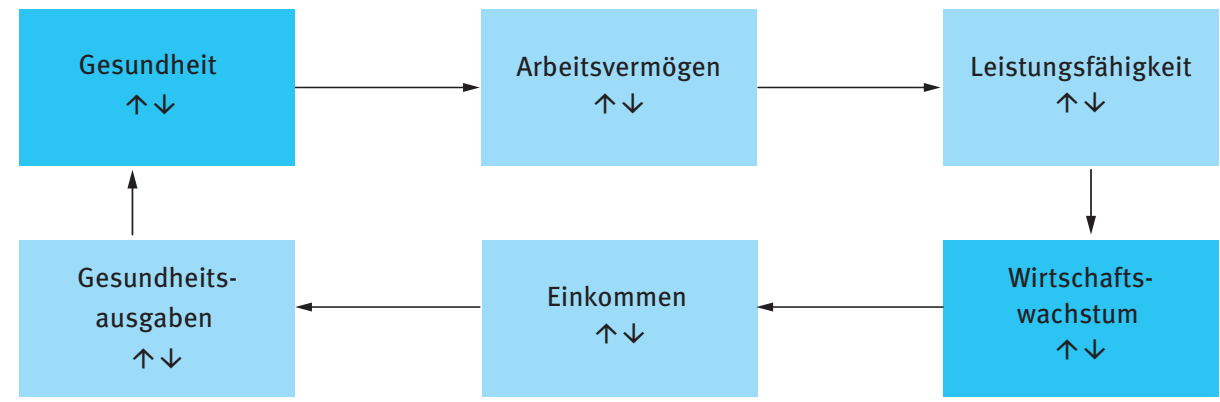

Abb. 19.6: Zusammenhang von Gesundheit und Wirtschaftswachstum. $\uparrow$ steigt, $\downarrow$ sinkt. 


\subsubsection{Schutz vor finanziellen Folgen von Krankheit}

Alle Mitgliedstaaten der Vereinten Nationen haben sich im Rahmen der Ziele für nachhaltige Entwicklung (Sustainable Development Goals; s. Kap. 7.3) geeinigt darauf hinzuwirken, bis 2030 eine allgemeine Gesundheitsversorgung (Universal Health Coverage; s. Kap. 18.2) zu erreichen. Allgemeine Gesundheitsversorgung beinhaltet, dass alle Menschen benötigte gesundheitsfördernde, präventive, heilende, rehabilitierende und palliative Gesundheitsleistungen von ausreichender Qualität nutzen können, ohne dadurch in finanzielle Schwierigkeiten zu geraten [40].

Um das Ziel des Schutzes vor finanziellen Krankheitsfolgen zu erreichen, sollte die Finanzierung von Gesundheitsleistungen möglichst unabhängig von Selbstzahlungen und Zuzahlungen aus eigener Tasche im Krankheitsfall sein. Statt Selbstzahlungen im Bedarfsfall sollten Finanzierungsmechanismen gestärkt werden, die auf Vorauszahlung und gegenseitiger Absicherung beruhen. Dies bedeutet, gesunde Menschen sollen dazu angehalten werden durch Besteuerung und/oder Versicherungsbeiträge, eine Vermögensrücklage für die Bezahlung von Gesundheitsleistungen für sich und, bei einer Solidargemeinschaft, auch für Andere aufzubauen. Im Falle einer Erkrankung wird die medizinische Behandlung aus dieser Vermögensrücklage bezahlt. Ob Steuern erhoben werden, um Gesundheitsleistungen zu finanzieren, oder zweckgebundene Beiträge in eine staatliche oder private Krankenversicherung fließen oder Mischformen genutzt werden, wird in unterschiedlichen Ländern unterschiedlich gehandhabt. Grundsätzlich können sowohl gesetzliche (steueroder beitragsfinanziert) als auch private Versicherungssysteme ihre Versicherten vor den finanziellen Folgen von Krankheit durch die Verteilung des finanziellen Risikos auf eine Gruppe (von Gesunden) oder auf einen längeren Zeitraum (die gesamte Zeit potentieller Erwerbsfähigkeit) bewahren.

Es gibt unterschiedliche Schätzungen über die Höhe der finanziellen Mittel, die erforderlich wären, um dem Ziel näherzukommen allen Menschen auf der Welt, einen Zugang zu benötigten medizinischen Maßnahmen zu ermöglichen ohne in eine finanzielle Notlage zu geraten. Empfehlungen liegen bei mindestens 5 \% des Bruttoinlandsprodukts und für Länder mit niedrigem Einkommen zusätzlich bei absoluten Gesundheitsausgaben von 86 internationalen Dollar pro Kopf und Jahr bei 2015 Preisen [41]. Im Jahr 2019 gaben 60 von 194 Volkswirtschaften mit verfügbaren Daten weniger als $5 \%$ des Bruttoinlandsprodukts oder weniger als 86 internationale Dollar von 2019 pro Kopf. Aus Sicht eines einzelnen Menschen ist nach Schätzungen ein persönliches Einkommen von mindestens 10,89 internationalen Dollar von 2011 notwendig, um Zugang zu grundlegenden medizinischen Leistungen zu erhalten [42].

In Deutschland, wo das weltweit älteste soziale Krankenversicherungssystem 1883 per Gesetz eingeführt wurde, beliefen sich die Gesundheitsausgaben im Jahr 2019 auf 11,4 \% des Bruttoinlandsprodukts und weniger als 0,1 \% der Bevölkerung waren nicht krankenversichert [43]. Nach Einführung der verpflichtenden Krankenversicherung für etwa $10 \%$ der Bevölkerung (zunächst nur für Arbeiter mit einem 
Jahreseinkommen von unter 2000 Mark) wurde das Krankenversicherungssystem in Deutschland schrittweise zu einer universellen Krankenversicherung ausgebaut. Seit den 1960er Jahren besteht ein Versicherungssystem, in dem gesetzliche und private Krankenversicherungen, gemeinsam mit weiteren Sozialversicherungen und steuerfinanzierten Gesundheitsleistungen, für mehr als $90 \%$ der Bevölkerung in Deutschland einen großen Teil der Gesamtausgaben für Gesundheit (88 \% in 2019) finanzieren $[44,45]$. In Ländern mit niedrigen oder mittleren Einkommen können Mikrokrankenversicherungen, die gezielt deren große Bevölkerungsteile im informellen Arbeitssektor versichern, eine Stärkung der Sozialversicherungssysteme ergänzen und zur Absicherung von Menschen mit geringem Einkommen gegen finanzielle Risiken im Krankheitsfall beitragen (s. Kap. 18.2.3 und [46,47]).

Neben der Versicherung und Vorausbezahlung von Gesundheitsausgaben trägt zum Schutz vor finanziellen Krankheitsfolgen bei, wenn Gesundheitsausgaben einen hohen Gegenwert im Vergleich zu ihren Kosten schaffen. Während sich die globale Gesundheit in den vergangenen Jahrzehnten zum Teil erheblich verbessert hat, wird zugleich geschätzt, dass 20-40 \% der weltweiten Gesundheitsausgaben verschwendet sein könnten, also sich nicht in einer Verbesserung der Gesundheit niederschlagen [33]. Im nächsten Kap. 20 zu ökonomischer Bewertung in der globalen Gesundheit wird eine strukturierte Vorgehensweise für den Vergleich der Nutzen und Kosten von Gesundheitsmaßnahmen besprochen, die dazu beitragen kann, die Wirksamkeit und Wirtschaftlichkeit von Gesundheitsausgaben zu steigern.

\subsection{Kernaussagen}

1. Ein internationaler Vergleich von Lebenserwartung und Bruttoinlandsprodukt pro Kopf deutet auf einen starken Zusammenhang zwischen Wohlstand und Gesundheit hin. Historisch betrachtet haben Gesundheit und Wohlstand weltweit zugenommen. Durch medizinisch-technischen Fortschritt waren und sind Verbesserungen bei der Gesundheit auch unabhängig von einer Änderung des Wohlstandsniveaus möglich.

2. Die stärkste Zunahme von Gesundheit und Wohlstand fand in der Vergangenheit in Ländern statt, die nun zu den Ländern mit hohem Einkommen zählen. Länder der niedrigeren und mittleren Einkommensgruppen haben ein großes Aufholpotential, aufgrund von medizinisch-technischem Fortschritt voraussichtlich sogar in kürzeren Zeiträumen als in der Vergangenheit beobachtet.

3. Das Ausmaß in dem mehr Wohlstand oder eine gleichere Verteilung von Wohlstand zu mehr Gesundheit führen ist schwer bestimmbar. Der beobachtete starke Zusammenhang zwischen Indikatoren, die Gesundheit und Wohlstand abschätzen, legt allerdings nahe, dass Armutsbekämpfung und die Verbesserung der Gesundheit auf Bevölkerungsebenen im Allgemeinen sich gegenseitig ergänzende Ziele sind. 
4. Nach einer Zunahme der Einkommensungleichheit, welche die Welt zwischen den 1960er und 1980er Jahren in eine Gruppe von ärmeren Ländern und eine Gruppe von wohlhabenderen Ländern zweiteilte, nahm die globale Einkommensungleichheit, geleitet vom wirtschaftlichen Wachstum in den bevölkerungsreichsten Staaten China und Indien, seit etwa den 1990er Jahren bis zum Beginn der COVID-19-Pandemie im Jahr 2020 ab. Die Einkommensungleichheit innerhalb von Ländern hat innerhalb der letzten beiden Jahrzehnte teilweise zugenommen. Deshalb werden mit zunehmender Häufigkeit größere Unterschiede innerhalb eines Landes als zwischen Ländern beobachtet.

5. Ärmere Menschen sind häufig mehrfachen Risiken durch Krankheit ausgesetzt. Zum einen haben sie ein erhöhtes Risiko zu erkranken, zum anderen haben sie höheres Risiko, eine Behandlung im Krankheitsfall selbst bezahlen zu müssen und damit durch einen Krankheitsfall in eine finanzielle Notlage zu geraten. Gesundheitssysteme, in denen Gesundheitsausgaben durch Krankenversicherungsbeiträge oder Steuereinnahmen bezahlt werden, schützen vor finanziellen Notlagen durch Krankheit und verbessern dadurch den Zugang zu Gesundheitsversorgung. Insbesondere, aber nicht nur in Ländern mit niedrigem oder niedrigem mittlerem Einkommen stellt der Auf- und Ausbau einer alle Menschen einschließenden und vor finanzieller Notlage im Krankheitsfall schützenden Gesundheitsausgabenfinanzierung eine wichtige und aktuelle Aufgabe dar.

\subsection{Fragen}

- Welcher Zusammenhang besteht zwischen Lebenserwartung und Wohlstand, gemessen als Bruttoinlandsprodukt pro Kopf, wenn Lebenserwartung und Wohlstand zwischen Ländern verglichen werden?

- Wie wird der Zusammenhang zwischen Lebenserwartung und Wohlstand durch medizinisch-technischen Fortschritt verändert?

- Welche Faktoren haben in der Vergangenheit am meisten zu Verlängerung der durchschnittlichen Lebenserwartung beigetragen?

- Welche unterschiedlichen Erklärungsansätze gibt es für den beobachteten Zusammenhang zwischen Lebenserwartung und Wohlstand? Benennen und erklären Sie diese zunächst. Diskutieren Sie danach inwieweit Sie einen oder mehrere dieser Erklärungsansätze in dem folgenden Satz aus der Strategie der Bundesregierung zur globalen Gesundheit wieder finden: „Gesundheit ist nicht nur eine Voraussetzung für Wohlstand, sozialen Zusammenhalt und gesellschaftliche Teilhabe, sondern auch Ergebnis und Indikator für die soziale, wirtschaftliche und ökologische Dimension nachhaltiger Entwicklung. “

- Welche Bedeutung haben die Begriffe Kausalzusammenhang, umgekehrter Kausalzusammenhang und Korrelation ohne Kausalzusammenhang? Erklären Sie diese Begriffe am Beispiel der beobachteten Beziehung zwischen Lebenserwar- 
tung und Wohlstand oder anhand von Überlegungen zu Einkommensungleichheit und Gesundheit.

- Wie hat sich die globale Einkommensungleichheit seit Anfang des 19. Jahrhunderts entwickelt?

- Wie hat sich der Einfluss von innerstaatlicher und zwischenstaatlicher Einkommensungleichheit auf die globale Einkommensungleichheit im Laufe der Zeit verändert?

- Wie verteilen die Bevölkerung, Gesundheitsausgaben und Krankheitslast im weltweiten Vergleich? Benutzen Sie für Ihre Beschreibung die Ländereinteilung der Weltbank.

- Wie könnte eine nicht endende Folge unangenehmer, einander bedingender Geschehnisse aussehen durch die Krankheit zur Armutsfalle wird? Beschreiben Sie beispielhaft einen Kreislauf für einen einzelnen Menschen oder für ein ganzes Land.

- Welche Arten der Finanzierung von Gesundheitsausgaben bieten einen Schutz vor finanziellen Folgen von Krankheit? Diskutieren den möglichen Schutzmechanismus sowie Stärken und Schwächen folgender Träger von Gesundheitsausgaben: öffentliche Haushalte, Sozialversicherungen, private Versicherungen, sonstige private Organisationen (z. B. Nichtregierungsorganisationen), Selbstzahlende im Krankheitsfall, Geberländer von Entwicklungshilfe für Gesundheit.

\section{Danksagung}

Till Bärnighausen wurde von der Alexander von Humboldt-Stiftung durch eine vom Bundesministerium für Bildung und Forschung geförderte Alexander von HumboldtProfessur unterstützt.

\section{Literatur}

Letzter Zugriff auf alle Internetquellen und Datenquellen: Oktober 2020.

[1] Preston SH. The Changing Relation between Mortality and Level of Economic Development. Population Studies 1975; 29: 231-248. doi:10.2307/2173509.

[2] Kontis V, Bennett JE, Mathers CD, u. a. Future life expectancy in 35 industrialised countries: projections with a Bayesian model ensemble. The Lancet 2017; 389: 1323-1335. doi:10.1016/ S0140-6736(16)32381-9.

[3] Denzer A, Weichelt C. Eine Navigationshilfe im Dschungel der Länderklassifizierungen. KFW Fokus Entwicklungspolitik2 2014; 5: 1-3.

[4] Pingali PL. Green Revolution: Impacts, limits, and the path ahead. Proceedings of the National Academy of Sciences 2012; 109: 12302-12308. doi:10.1073/pnas.0912953109.

[5] Roser M, Ortiz-Ospina E, Ritchie H. Mortality and life expectancy by age. Published online at OurWorldInData.org, https://ourworldindata.org/life-expectancy\#mortality-and-life-expectancy-by-age.

[6] Bundeszentrale für politische Bildung. Deutsche Verhältnisse. Eine Sozialkunde: Bevölkerung: Historischer Rückblick, https://www.bpb.de/politik/grundfragen/deutsche-verhaeltnisse-einesozialkunde/. 
[7] de la Croix D, Licandro 0. The longevity of famous people from Hammurabi to Einstein. Journal of Economic Growth 2015; 20: 263-303. doi:10.1007/s10887-015-9117-0.

[8] World Population Prospects 2019: Data Booklet (ST/ESA/SER.A/424). New York, NY: United Nations, Department of Economic and Social Affairs, Population Division, 2019.

[9] Kohler S. Die Vermessung der globalen Gesundheit. Gesundheit und Gesellschaft / Wissenschaft 2019; 19: 16-23.

[10] Cassini A, Högberg LD, Plachouras D, u. a. Attributable deaths and disability-adjusted life-years caused by infections with antibiotic-resistant bacteria in the EU and the European Economic Area in 2015: a population-level modelling analysis. The Lancet Infectious Diseases 2019; 19: 56-66. doi:10.1016/S1473-3099(18)30605-4.

[11] Foreman KJ, Marquez N, Dolgert A, u. a. Forecasting life expectancy, years of life lost, and allcause and cause-specific mortality for 250 causes of death: reference and alternative scenarios for 2016-40 for 195 countries and territories. The Lancet 2018; 392: 2052-2090. doi:10.1016/ S0140-6736(18)31694-5.

[12] Bor J, Herbst AJ, Newell M-L, u. a. Increases in adult life expectancy in rural South Africa: valuing the scale-up of HIV treatment. Science 2013; 339: 961-965. doi:10.1126/science.1230413.

[13] Reniers G, Blom S, Calvert C, u. a. Trends in the burden of HIV mortality after roll-out of antiretroviral therapy in KwaZulu-Natal, South Africa: an observational community cohort study. The Lancet HIV 2016; S533-S542. doi:10.1016/S2352-3018(16)30225-9.

[14] Jetter M, Laudage S, Stadelmann D. The Intimate Link Between Income Levels and Life Expectancy: Global Evidence from 213 Years. Social Science Quarterly 2019; 100: 1387-1403. doi:10.1111/ssqu.12638.

[15] Imhof AE (Hrsg). Lebenserwartungen in Deutschland, Norwegen und Schweden im 19. und 20. Jahrhundert. Berlin: Akademie Verlag, 1994.

[16] Pritchett L, Summers LH. Wealthier is healthier. The Journal of Human Resources 1996; 31: 841868. doi:10.2307/146149.

[17] Strategie der Bundesregierung zur globalen Gesundheit: Verantwortung - Innovation - Partnerschaft: Globale Gesundheit gemeinsam gestalten. Berlin: Bundesministerium für Gesundheit, Referat Globale Gesundheitspolitik, 2020.

[18] Bourguignon F. The Globalization of Inequality. Princeton, New Jersey, USA: Princeton University Press, 2015.

[19] Roser M. Global Economic Inequality. Published online at OurWorldInData.org, https://ourworldindata.org/global-economic-inequality.

[20] Hellebrandt T, Mauro P. The Future of Worldwide Income Distribution. Washington DC: Peterson Institute for International Economics, 2015.

[21] Poverty and Shared Prosperity 2020: Reversals of Fortune. Washington DC: World Bank, 2020. doi:10.1596/978-1-4648-1602-4.

[22] Bourguignon F, Morrisson C. Inequality Among World Citizens: 1820-1992. American Economic Review 2002; 92: 727-744. doi:10.1257/00028280260344443.

[23] Kanbur R, Wang Y, Zhang X. The great Chinese inequality turnaround. Journal of Comparative Economics. doi:10.1016/j.jce.2020.10.001.

[24] Lustig N, Lopez-Calva LF., Ortiz-Juarez E. Deconstructing the Decline in Inequality in Latin America. In: Basu K, Stiglitz I (Hrsg) Inequality and Growth: Patterns and Policy: Volume II: Regions and Regularities. London: Palgrave McMillan, 2016, S. 212-247.

[25] Measuring the 1\%: Economists are rethinking the numbers on inequality. The Economist, 2019.

[26] Tomaskovic-Devey D, Rainey A, Avent-Holt D, u. a. Rising between-workplace inequalities in high-income countries. Proceedings of the National Academy of Sciences 2020; 117: 9277-9283. doi:10.1073/pnas.1918249117. 
[27] Lynch JW. Income inequality and mortality: importance to health of individual income, psychosocial environment, or material conditions. BMJ 2000; 320: 1200-1204. doi:10.1136/ bmj.320.7243.1200.

[28] Marmot M. Psychosocial and material pathways in the relation between income and health: a response to Lynch et al. BMJ 2001; 322: 1233-1236. doi:10.1136/bmj.322.7296.1233.

[29] Falk A, Kosse F, Menrath I, u. a. Unfair Pay and Health. Management Science 2018; 64: 14771488. doi:10.1287/mnsc.2016.2630.

[30] Pickett KE, Wilkinson RG. Income inequality and health: a causal review. Social Science and Medicine 2015; 128: 316-326. doi:10.1016/j.socscimed.2014.12.031.

[31] Herman D, Afulani P, Coleman-Jensen A, u. a. Food insecurity and cost-related medication underuse among nonelderly adults in a nationally representative sample. American Journal of Public Health 2015; 105: e48-e59. doi:10.2105/AJPH.2015.302712.

[32] Hall RE, Jones $\mathrm{Cl}$. The Value of Life and the Rise in Health Spending. The Quarterly Journal of Economics 2007; 122: 39-72. doi:10.1162/qjec.122.1.39.

[33] Global Health Expenditure Atlas. Geneva: World Health Organization, 2014.

[34] Financing Global Health 2019: Tracking Health Spending in a Time of Crisis. Seattle, WA: Institute for Health Metrics and Evaluation, 2020.

[35] Chang AY, Cowling K, Micah AE, u. a. Past, present, and future of global health financing: a review of development assistance, government, out-of-pocket, and other private spending on health for 195 countries, 1995-2050. The Lancet 2019; 393: 2233-2260. doi:10.1016/S0140-6736 (19)30841-4.

[36] McIntyre D, Thiede M, Dahlgren G, u. a. What are the economic consequences for households of illness and of paying for health care in low- and middle-income country contexts? Social Science and Medicine 2006; 62: 858-865. doi:10.1016/j.socscimed.2005.07.001.

[37] Arsenijevic J, Pavlova M, Rechel B, u. a. Catastrophic health care expenditure among older people with chronic diseases in 15 European countries. PLOS ONE 2016; 11: e0157765. doi:10.1371/journal.pone.0157765.

[38] Xu K, Evans DB, Carrin G, u. a. Protecting Households From Catastrophic Health Spending. Health Affairs 2007; 26: 972-983. doi:10.1377/hlthaff.26.4.972.

[39] Xu K, Evans DB, Kawabata K, u. a. Household catastrophic health expenditure: a multicountry analysis. The Lancet 2003; 362: 111-117. doi:10.1016/S0140-6736(03)13861-5.

[40] World Health Organization. Universal health coverage and health financing, https://www.who. int/health_financing/universal_coverage_definition/.

[41] Mcintyre D, Meheus F, Røttingen J-A, u. a. What level of domestic government health expenditure should we aspire to for universal health coverage? Health Economics, Policy and Law 2017; 12: 125-137. doi:10.1017/S1744133116000414.

[42] Sterck O, Roser M, Ncube M, u. a. Allocation of development assistance for health: is the predominance of national income justified? Health Policy and Planning 2018; 33: i14-i23. doi:10.1093/heapol/czw173.

[43] Sozialleistungen: Angaben zur Krankenversicherung (Ergebnisse des Mikrozensus) 2019. In: Fachserie 13, Reihe 1.1. Wiesbaden: Statistisches Bundesamt, 2020.

[44] Busse R, Blümel M, Knieps F, u. a. Statutory health insurance in Germany: a health system shaped by 135 years of solidarity, self-governance, and competition. The Lancet 2017; 390: 882897. doi:10.1016/S0140-6736(17)31280-1.

[45] Bärnighausen T, Sauerborn R. One hundred and eighteen years of the German health insurance system: are there any lessons for middle- and low-income countries? Social Science \& Medicine 2002; 54: 1559-1587. doi:10.1016/S0277-9536(01)00137-X.

[46] Dror DM. Microinsurance: A short history. International Social Security Review 2019;72:107126. doi:10.1111/issr.12223. 
[47] Dror DM. Health Microinsurance Programs in Developing Countries. In: Culyer AJ (Hrsg) Encyclopedia of Health Economics. Elsevier, 2014, S. 412-421.

\section{Verzeichnis der ausgewerteten Datenquellen}

Financing Global Health Database: https://ghdx.healthdata.org/series/financing-global-health-fgh Data combined by Gapminder: https://www.gapminder.org/data/documentation/ Global Burden of Disease Study 2019 Data Resources: https://ghdx.healthdata.org/gbd-2019 World Bank Country and Lending Groups: https://data.worldbank.org/about/country-and-lending-groups World Development Indicators: https://datatopics.worldbank.org/world-development-indicators/

\section{Links zu Visualisierungen von globalen Gesundheitsdaten}

Exemplars in Global Health Data Explorer: https://www.exemplars.health/data-explorer/ Gapminder Dollar Street: https://www.gapminder.org/dollar-street/

Gapminder Tools: https://www.gapminder.org/tools/

Global Health Expenditure Database: https://apps.who.int/nha/database

Global Health Financing Database: https://vizhub.healthdata.org/fgh/

Global Burden of Disease Studie: https://vizhub.healthdata.org

Our World in Data: https://ourworldindata.org

Sustainable Development Goals Tracker: https://sdg-tracker.org/ United Nations World Population Prospects: https://esa.un.org/unpd/wpp/

WHO Global Health Expenditure Database https://apps.who.int/nha/database WHO Global Health Observatory: https://www.who.int/data/gho World Bank Open Data: https://data.worldbank.org/ World Bank Poverty \& Equity Data Portal: http://povertydata.worldbank.org/ 
